\title{
FAST BUS PROTECTION USING IEC 61850
}

\section{By}

\author{
Cassandra Ha Goff
}

Approved:

Ahmed H. Eltom

Professor of Electrical Engineering (Chair)

Abdul R. Ofoli

Assistant Professor of Electrical Engineering (Committee Member)
Stephen D. Craven

Adjunct Professor of Electrical Engineering (Committee Member)

Gary L. Kobet

Adjunct Professor of Electrical Engineering (Committee Member)
A. Jerald Ainsworth
Dean of the Graduate School

William H. Sutton

Dean of the College of Engineering and Computer Science 


\section{FAST BUS PROTECTION USING IEC 61850}

By

\section{Cassandra Ha Goff}

A Thesis Submitted to the Faculty of the University

of Tennessee at Chattanooga in Partial

Fulfillment of the Requirements of the

Degree of Master of Science

in Electrical Engineering

The University of Tennessee at Chattanooga

Chattanooga, Tennessee

May 2013 


\begin{abstract}
International Electrotechnical Commission (IEC) 61850 is the latest technology that allows electric power utilities to build low cost digital substations. Because limited objective information is available, electric power utilities desire independent evaluations of IEC 61850 from end users.

The objective of this study was to compare the IEC 61850 method to the hardwired method to find an optimal solution for the fast bus protection scheme. System disturbance clearing time, engineering and construction costs, and interoperability between multivendor microprocessor-based relays were used as the criteria for the evaluation.

This research measured the protection speed of the hardwired method and the IEC 61850 method for the fast bus protection scheme, utilizing relays from the same and mixed manufacturers. The results of the study show that IEC 61850 method is simple, cost effective, and offers speed of operation comparable to the hardwired method.
\end{abstract}




\section{TABLE OF CONTENTS}

LIST OF TABLES

LIST OF FIGURES

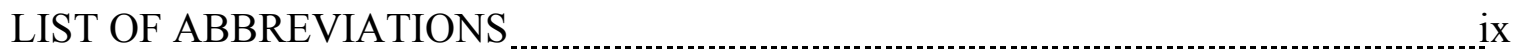

\section{CHAPTER}

1. INTRODUCTION

2. LITERATURE REVIEW

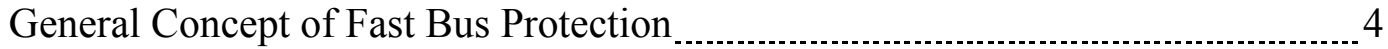

Fast Bus Protection Scheme Using the Traditional Hardwired Method _................ 5

Fast Bus Protection Scheme Using IEC 61850 Communications Protocol ............... 6

Logical Node $\ldots$

GOOSE Messages $\ldots \ldots \ldots \ldots$

Multicast Messages $\ldots \ldots$

Time Allowed To Live $\quad 10$

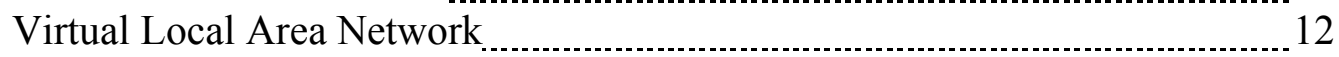

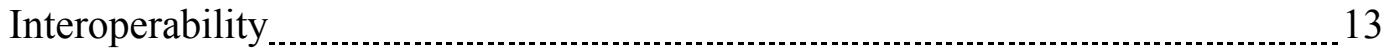

3. PRIOR ART $\ldots$

4. RELAY CONFIGURATIONS

Fast Bus Protection Scheme Using the Traditional Hardwired Method _............... 17

Fast Bus Protection Scheme Using IEC 61850

Using Microprocessor-based Relays from Same Manufacturer _..................... 19

GOOSE Messages from the Publisher - Feeder 1 Relay (Vendor A) _.........20

GOOSE Messages for the Subscriber - Bus Relay _.............................21

Using Microprocessor-based Relays from Different Manufacturers ................22

GOOSE Messages from the Publisher - Feeder 1 Relay (Vendor B) _........ 22

5. TEST PROCEDURES AND RESULTS _._.

Test Procedures $\ldots \ldots \ldots$

The Hardwired Method 
Testing Using High Ratios of Multiples of Pickup of Settings _.................... 28

Testing Using Low Ratios of Multiples of Pickup of Settings _..................... 28

IEC 61850 GOOSE Messaging Method Using Same Relay Manufacturers _..........28

IEC 61850 GOOSE Messaging Method Using Different Relay Manufacturers _... 29

Test Results

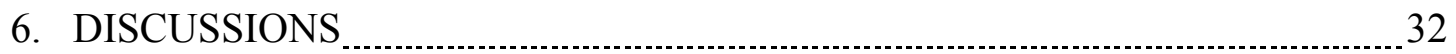

System Disturbance Clearing Time

Average Time Latency of GOOSE Messages $\ldots \ldots \ldots \ldots \ldots \ldots$

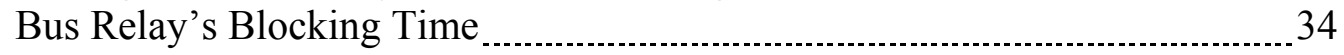

Interoperability of IEC $61850 \ldots$

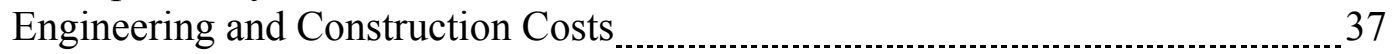

7. CONCLUSIONS

REFERENCES

VITA 


\section{LIST OF TABLES}

5.1 Ground Faults' Blocking Speeds Using High Ratios of Multiples of Pickups

5.2 Phase Faults' Blocking Speeds Using High Ratios of Multiples of Pickups 30

5.3 Ground Faults' Blocking Speeds Using Low Ratios of Multiples of Pickups. 30

5.3 Phase Faults' Blocking Speeds Using Low Ratios of Multiples of Pickups 31 


\section{LIST OF FIGURES}

2.1 One Line Diagram of a Fast Bus Protection Scheme $\ldots$

2.2 Hardwired Fast Bus Protection Scheme for Two Feeders _................................... 5

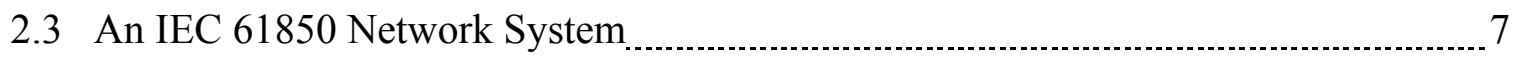

2.4 Functional Modeling of Data in IEC 61850 8

2.5 Logical Node for an IOC Element in IEC 61850

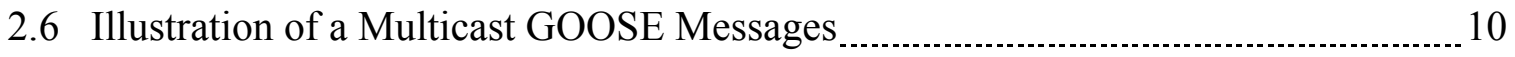

2.7 Event-Driven Real Time Communication with GOOSE

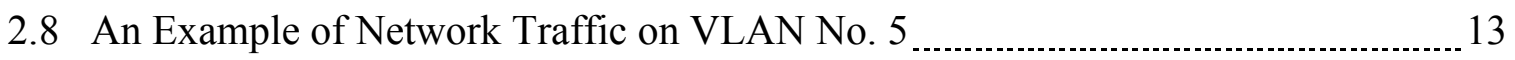

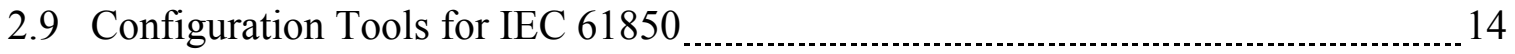

4.1 Fast Bus Protection Scheme for Feeder 1 _......................................... 17

4.2 Fast Bus Protection Scheme for One Feeder Using IEC 61850

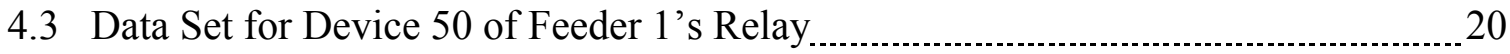

4.4 GOOSE Transmit Message for a Publisher _ 21

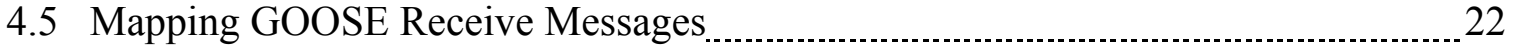

4.6 Mapping GOOSE Transmit Messages for a Publisher …… 23

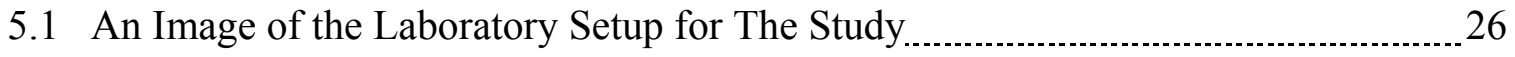

6.1 GOOSE Capturing Time Using the Omicron Test Set and Relay Specifications ...... 33

6.2 The Actual GOOSE Blocking Speed Versus Hardwired Speed for Ground Faults 35

6.3 The Actual GOOSE Blocking Speed Versus Hardwired Speed for Phase Faults _..... 36 
6.4 Pictures of Construction Using a Hardwired Method and an IEC 61850 Method 38 


\section{LIST OF ABBREVIATIONS}

50G1P, Instantaneous Overcurrent Pickup Setting of Residual Ground Relay Element 50P1P, Instantaneous Overcurrent Pickup Setting of Phase Relay ElementCT, Instrument Current Transformer

EM, Electromechanical Relay

GOOSE, Generic Object Oriented Substation Event

GSSE, Generic Substation State Events

IEC, International Electrotechnical Commission

IEC 61850, IEC 61850 Communications Protocol

IED, Intelligent Electronic Device

IOC, Instantaneous Overcurrent relay elementLAN, Local Area Network

LN, Logical Node

NC, Normally Closed Relay Output Contact

NIOC, Neutral Instantaneous Overcurrent relay element

NO, Normally Open Relay Output Contact

OUTi, Abbreviation for a relay Output Contact

SEL, Schweitzer Engineering Laboratories, Inc.

UCA, Utility Communication Architecture

VLAN, Virtual Local Area Network

VB00i, Relay Virtual Bit numbered $i^{\text {th }}$ 


\section{CHAPTER 1}

\section{INTRODUCTION}

Over the last decade, the power industry has been facing massive changes. The advancement of computer technology, the global energy crisis, and the goal of producing clean energy have pressured the global power industry to maximize their operational efficiency to maintain low electric rates. Maintaining reliable power delivery at a lower cost has become the number one objective for electric power utilities.

Electric power utilities are searching for solutions to keep their electric rates low while still maintaining the quality of service. Retiring electromechanical (EM) relays and replacing them with microprocessor-based relays was one of the solutions in reducing the operating cost. This was because microprocessor-based relays offer extensive self-testing capabilities, detailed metering, and event reporting functions to lower utility dependence on routine maintenance testing. Because of these features, microprocessor-based relays are also referred to as intelligent electronic devices (IEDs). Also, the prices for microprocessor-based relays are much lower compared to the prices of EM relays. In brief, the technology of EM relays was surpassed by the technology of microprocessor-based relays.

Although microprocessor-based relays offer many advance features, calculating, applying, and testing microprocessor-based relays are much more complex compared to EM relays. Relay engineers and relay testers are required not only to have the power protection knowledge but also the computer knowledge to perform their work as well. It took the power 
industry more than a decade to accept and to be efficient working with microprocessor-based relays [1].

Most, if not all electric power utilities are now using microprocessor-based relays to protect their power systems. Electric power utilities now utilize the event report feature of the microprocessor-based relays for analyzing and locating faults instead of incurring the additional cost of installing digital fault recorders.

Because of the high cost and the increased requirements for maintenance, in many cases the bus differential protection scheme is not installed on the distribution or sub-transmission systems. As a result, bus faults are cleared by backup relays with longer fault clearing time, caused by the need for coordination between the distribution feeder relays and the transformer relays [2].

To reduce the installation costs of the current transformers (CTs) and the profusion of CT wiring without delaying bus fault clearing time, the fast bus protection scheme is implemented to replace the bus differential protection scheme $[3,4]$. The fast bus protection scheme is one of the simplest and least expensive schemes being used widely as a protection scheme for radial power distribution systems.

To respond more effectively to market changes and shorten asset downtime to protect revenue, the power industry is increasingly searching for the latest technological innovation in modernizing their infrastructure. IEC 61850 is the latest technology that was created to be the international standard of the communications protocol that allows power utilities to build lower cost digital substations. These substations use multivendor microprocessor-based relays that are connected to a local area network through Ethernet switches to perform substation automation, 
protection, monitoring, metering, and control using computer signals instead of the expensive hardwired copper circuitry.

Similarly to the beginning of the microprocessor-based relay era, electric power utilities are skeptical about the functionalities and the benefits compared to the negative effects or the hidden cost that IEC 61850 might bring. The question that the power utilities are asking is: Could the implementation of IEC 61850 actually provide a greater reduction in the installation, design, and maintenance cost?

The purpose of this study was to present an independent evaluation of the implementation of IEC 61850 on the fast bus protection scheme. The scope of this study was to compare the traditional hardwired method versus the IEC 61850 method for the fast bus protection scheme not the performance of relays between different manufacturers. The selection of relays was only based on available resources. The unbiased results of this study will be valuable and beneficial for the electric power distributors in determining if IEC 61850 is a viable replacement for the hardwired fast bus protection and other protection schemes as well. 


\section{CHAPTER 2}

\section{LITERATURE REVIEW}

\section{General Concept of Fast Bus Protection Scheme}

The fast bus protection scheme is also known as the bus zone interlock scheme. A bus fault is considered as an "in zone fault" while a feeder fault is considered as an "out of zone fault". The fast bus protection scheme consists of an overcurrent relay installed for each feeder that is used for the feeder protection and an overcurrent relay installed on the low side of a transformer that is used as a primary protection for a bus fault as shown in Figure 2.1 [5].

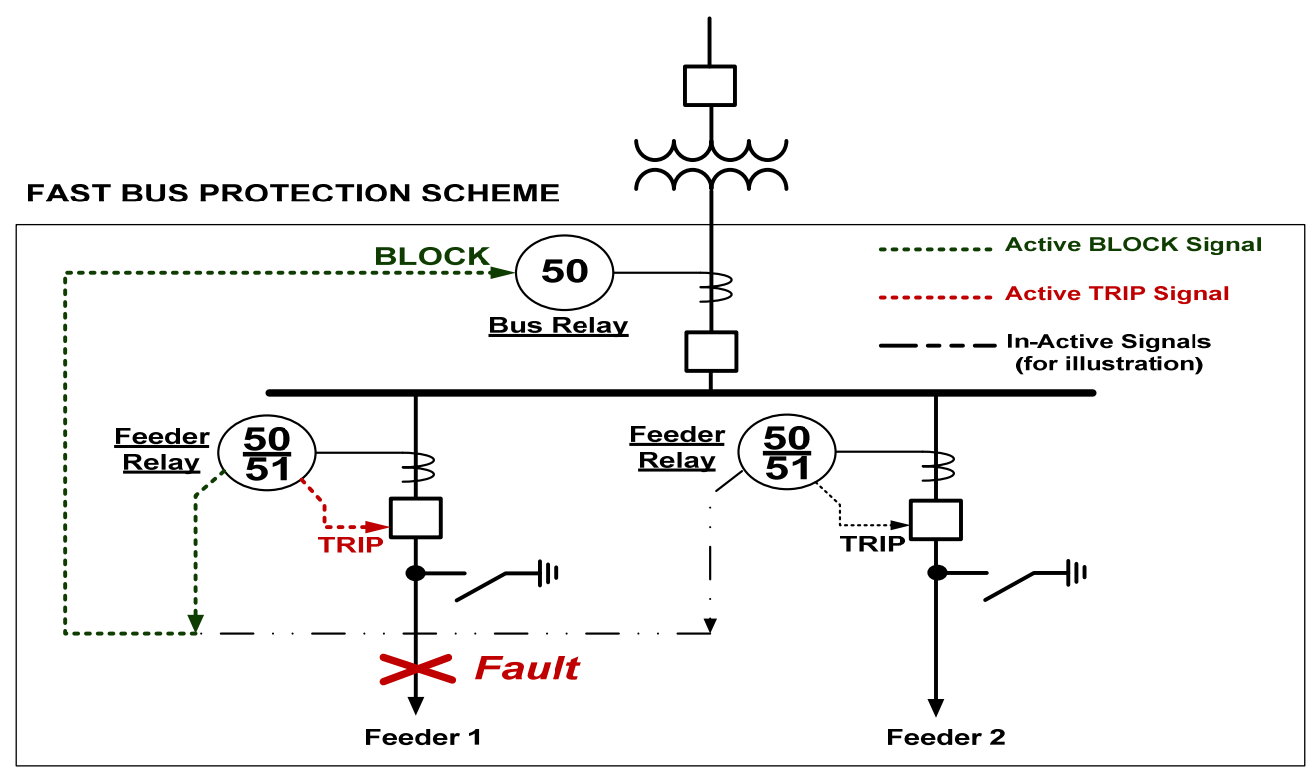

Fig 2.1 One Line Diagram of a Fast Bus Protection Scheme 
Fast Bus Protection Scheme Using the Traditional Hardwired Method

Due to cost savings, a minimum communication-aided trip that requires no additional purchase of communication equipment is normally implemented for the protection schemes in radial distribution systems. The fast bus protection scheme is implemented using that principle. The traditional hardwired fast bus protection scheme uses a relay output contact of the feeder relay to detect for the feeder faults, and a relay output contact of the bus relay to block for an out of zone fault as shown in Figure 2.2.

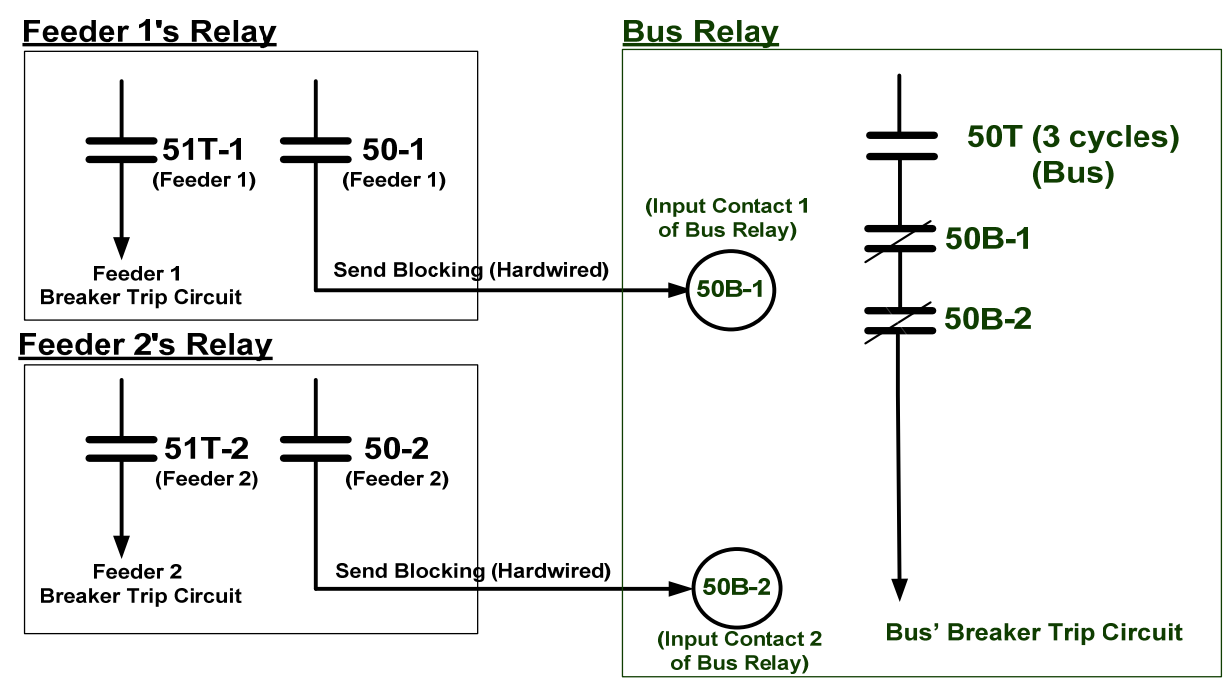

Fig 2.2 Hardwired Fast Bus Protection Scheme for Two Feeders

For EM relays, the bus relay's input contact coil (50B-1 or 50B-2) and its associated output contacts, as shown on the right of Figure 2.2, are supplied from an external relay (an auxiliary relay). The auxiliary relay used in this application has no protection function; it only consists of an input contact coil and a set of normally open (NO) or normally closed (NC) output contacts. The auxiliary relay is considered as a component of the bus relay. The position of these 
relay output contacts are toggled based on whether the relay input coil is energized or deenergized.

The fast bus protection scheme of microprocessor-based relays is implemented similarly to the fast bus protections scheme of EM relays. A relay output contact of the feeder relay is used to detect for the feeder faults. However, the same relay output contact of the feeder relay can also be used to block the bus relay from operating for an out of zone fault by software programming and physical connection. This will be addressed in more detail on page 18 of Chapter 4.

When a feeder fault occurs, the feeder relay's output contact (50-1 or 50-2), as shown on the left of Figure 2.2, is closed if the fault current is above its pickup setting. This action energizes the bus relay's input contact coil (50B-1 or 50B-2) which immediately causes the NC output contact (50B-1 or 50B-2) to open. The bus relay's output contact (50B-1 or 50B-2) stays opened which blocks the bus relay from tripping for an out of zone fault. A short timer (3 to 4 cycles) is applied to the bus relay (Device 50T) to avoid a race between the bus relay's output contact (50B-1 or 50B-2) opening and the bus relay's output contact (Device 50T) closing.

When a bus fault occurs, none of the feeder relays will measure any fault current; therefore, the bus relay's input contact coil (50B-1 or 50B-2) will not be energized. The bus relay's output contact (50B-1 or 50B-2), therefore, will not be toggled to an open position. The bus relay will operate as soon as the Device 50T times out.

Fast Bus Protection Scheme Using IEC 61850 Communications Protocol

IEC 61850 is a standard for the design of the substation automation and control communication system. IEC 61850 is a part of the International Electrotechnical Commission's 
(IEC) Technical Committee 57 (TC57) communication reference architecture for electric power systems [6]. IEC 61850 defines three communication paths: Process Bus, Station Bus (IED-toIED), and Client-to-Server communications.

For the scope of this study, only Station Bus and the communications protocol related to the fast bus protection scheme will be discussed. The principle of the fast bus protection scheme using IEC 61850 is the same as the principle of the hardwired application. However, the virtual high speed peer-to-peer (also termed as device-to-device or relay-to-relay) GOOSE messages are used to block the bus relay from tripping for an out of zone fault instead of the physical relay input and output contacts.

Before defining a GOOSE message, consider an IEC 61850 communications network between IEDs. An IEC 61850 network system is a system in which information is virtually exchanged between IEDs through an Ethernet network switch as illustrated in Figure 2.3 [7, 8, 9].

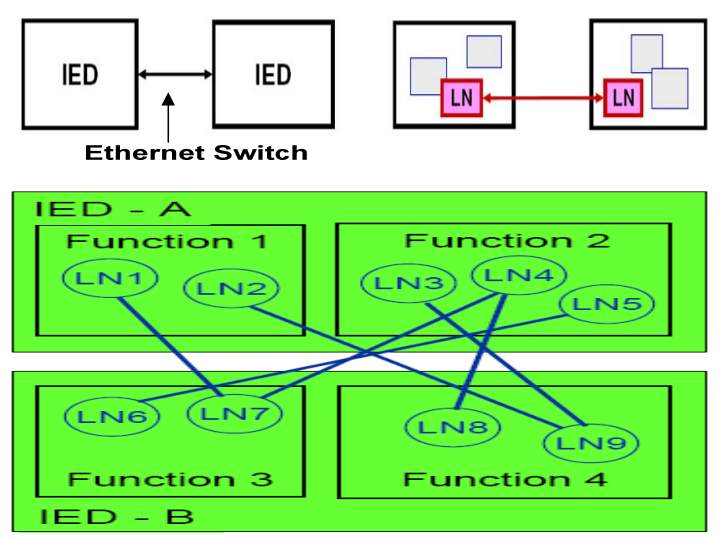

Logical Node Groups (First letter listed)

L System LN (2)

P Protection (28)

R Protection related (10)

C Control (5)

G Generic (3)

I Interfacing and archiving (4)

A Automatic control (4)

\section{Examples}

PDIF: Differential prolection

RBRF: Breaker failure

XCBR: Circuit breaker
M Metering and measurement (8)

$\mathrm{S}$ Sensor and monitoring (4)

$X$ Switchgear (2)

T Instrument transformers (2)

Y Power transformers (4)

Z Further power system equipment (15)

Fig 2.3 An IEC 61850 Network System 


\section{Logical Node}

Any function in an IED can be divided into sub-functions. Data are exchanged between functions and sub-functions residing in an IED. The smallest part of a function that exchanges data is called a logical node. Each logical node represents a function within a physical device as illustrated graphically in the lower left of Figure 2.3. The logic nodes in IEC 61850 are standardized to denote different functions in the substation automation system. The communication link between the logical nodes is called a logical connection. The IEC 61850 logical nodes and the communication of data between them are the core of interoperability [9].

As shown in Figure 2.4, an IED is modeled as a system of different functions that are built from the imaginary devices or logical nodes. The logical nodes are nothing more than the object-oriented programming of the functional data [10]. Peer-to-peer communications are used to perform protection, control, monitoring, and recording functions of IEDs.
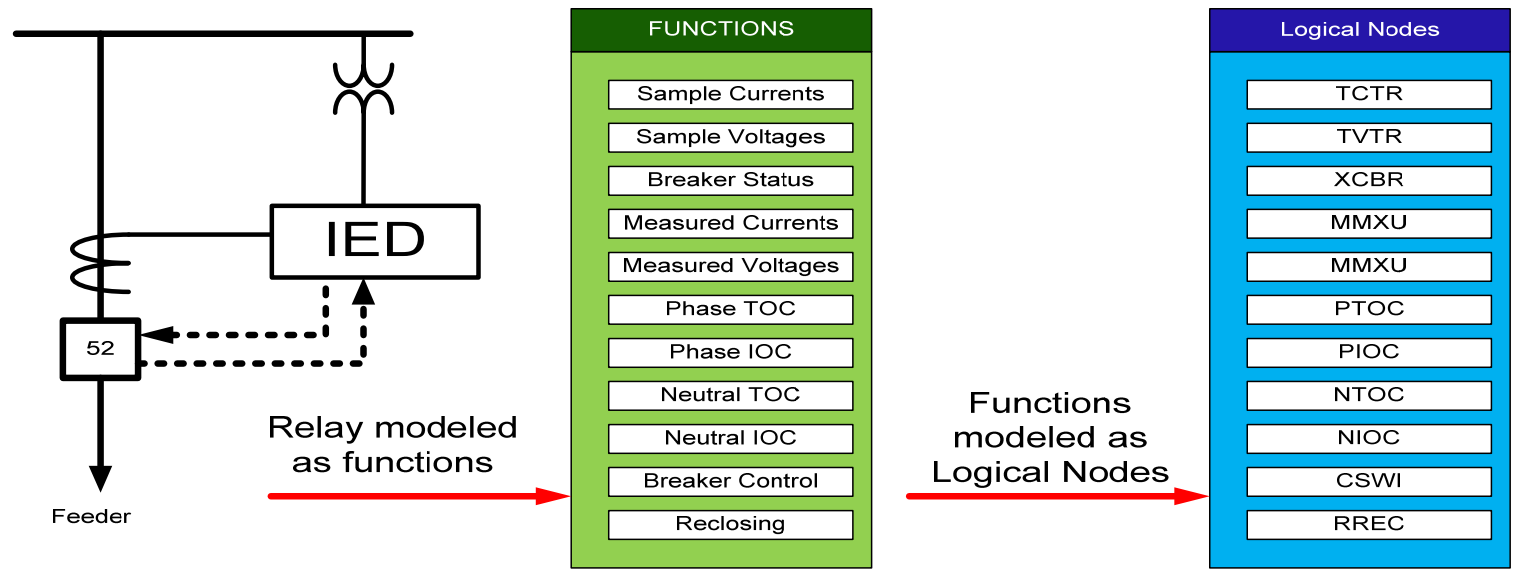

Fig 2.4 Functional Modeling of Data in IEC 61850 


\section{GOOSE Messages}

Each LN has a list of data objects with attributes. Each data object and its attribute together represent the information which needs to be exchanged among LNs by the communications service offered by IEC 61850. As illustrated in Figure 2.5 [7, 10], the data object of an instantaneous overcurrent relay (IOC) element and its attribute are the combined data that can be exchanged with other logical nodes. The interface of the communications services that the functional elements use is called the Abstract Communication Service Interface (ACSI). The Generic Object Oriented Substation Event (GOOSE) is one of the service models in the ASCI.
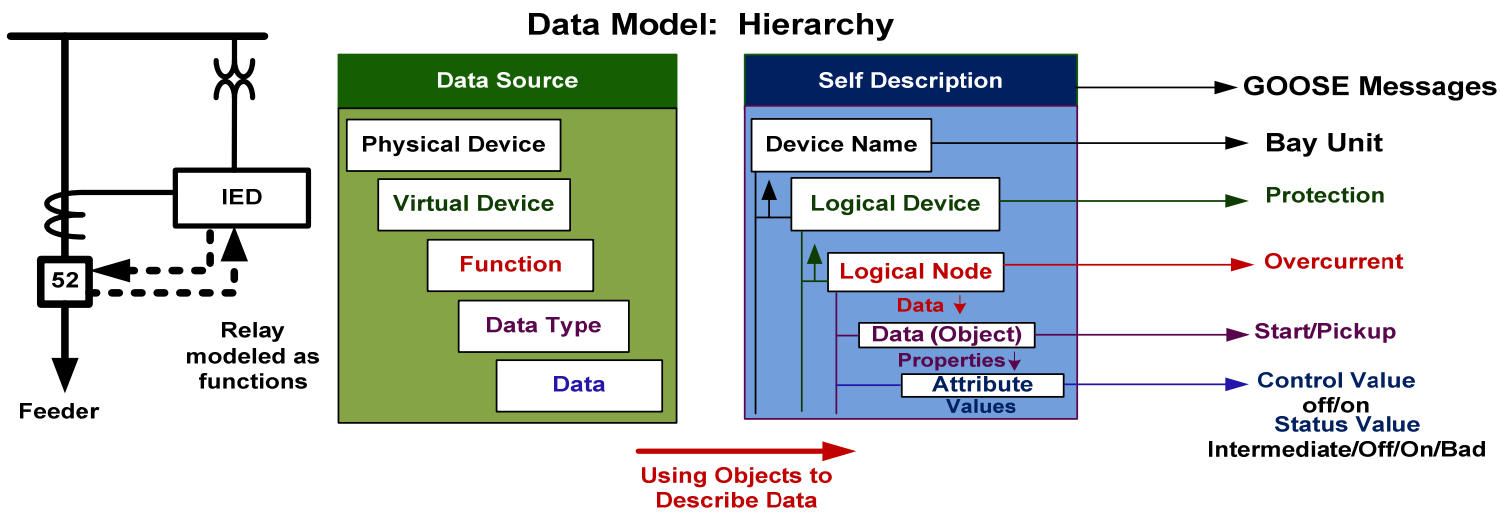

Fig 2.5 Logical Node for an IOC Element in IEC 61850

In summary, GOOSE message is a user-defined and a self-described set of data that is "published" on detection of a change in any of the contained data items. GOOSE message is event-driven and not published on one specific time interval [11]. Any device on the local area network that is interested in the published data can subscribe to the publisher GOOSE message as desired. GOOSE is known as a "Publisher-Subscriber message" [6]. The feeder relays are the 
publishers while the bus relay is the subscriber for the fast bus protection scheme as shown in Figure 2.1.

\section{Multicast Messages}

A GOOSE message is a reliable multicast message that is sent out from one source (publisher) to one or many destinations (subscribers). A multicast GOOSE message is illustrated as shown in Figure 2.6.

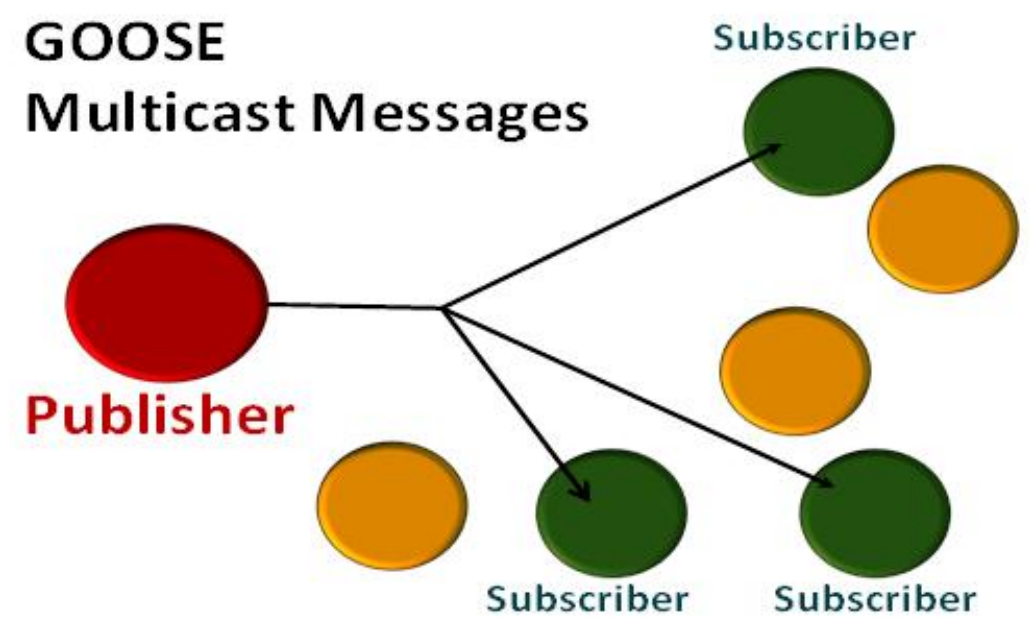

Fig 2.6 Illustration of Multicast GOOSE Messages

\section{Time Allowed To Live}

GOOSE messages contain information that allows the receiving devices (the subscribers) to detect a change and the time of the last change. A change in GOOSE messages could be a breaker position change or an analog measurement change in values of voltage, current, real power, reactive power, etc. The time of the last change allows the receiving devices to set the local timers relating to a given event. 
A "keep alive" message is periodically sent by the publisher to detect a potential failure. In the keep-alive message, there is a data set item which indicates the next GOOSE message will be sent in $T 0$ seconds, where $T 0$ is a user definable timer. If a subscriber fails to receive the messages in a specified time frame, an alarm can be set to indicate the failure of the publisher or the communication network. The IED can take an alternate action when this failure alarm occurs.

For digital input or output values, the GOOSE messages are sent based on the transition in the change of state from false-to-true or true-to-false. For analog measurements, the GOOSE messages are sent based on value changes greater than the configured deadband [12]. Figure 2.7 [13] illustrates the communication of GOOSE messages. The content of a GOOSE message and the maximum time $T 0$ (time allowed to live) are defined in a data set. When there is no event, the GOOSE messages are repeatedly sent with the maximum time interval T0. The GOOSE messages start immediately with the changed values in some short repetition interval (T1). The interval will be increased fast or slowly $(T 2, T 3)$ to the maximum time interval $T 0$ if there is no disturbance in the system.

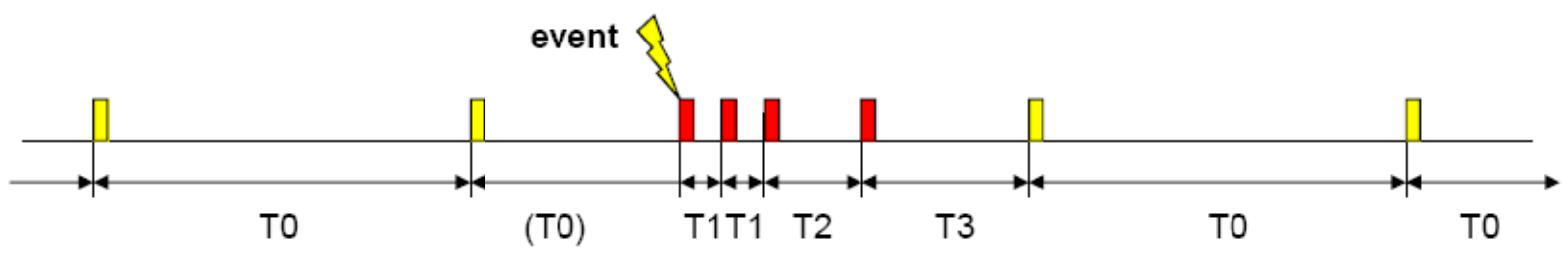

retransmission in stable conditions (no event for a long time).

T1 shortest retransmission time after the event.

T2, T3 retransmission times until achieving the stable conditions time. (the value of these times is an application issue)

Fig 2.7 Event-Driven Real Time Communication with GOOSE 
A GOOSE message must fit into a single Ethernet data frame which can be up to 1500 bytes. A typical GOOSE message is about 300 bytes long or 2400 bits. A well-designed IED can perform a detection of change with an average latency of 1 millisecond ( $\mathrm{ms}$ ) in the receiving device. Modern IEC 61850 implementations are able to send messages between protective relays at speeds between 1 to $2 \mathrm{~ms}$ [6].

\section{Virtual Local Area Network}

A Local Area Network (LAN) is a network that connects all the computer-based devices in a small area typically a single building or a group of buildings using an Ethernet switch, a router, fiber optic, or Wi-Fi networking connections.

A Virtual Local Area Network (VLAN) is a network that permits multiple logically separate LANs to reside on the physical network. VLAN allows devices in a LAN to be grouped by applications, logical function, or by applications without regard to physical location of the users.

Figure 2.8 illustrates the network traffic on VLAN No. 5 in which IED 1 is sending IEC 61850 GOOSE messages to other subscribers (IED 3, IED 4, IED 6, and IED 7). Note that the GOOSE messages from IED 1 did not reach IED 2 and IED 5 because they are on different VLANs. IED 2 is on VLAN No. 2 while IED 5 is on VLAN No. 7. 


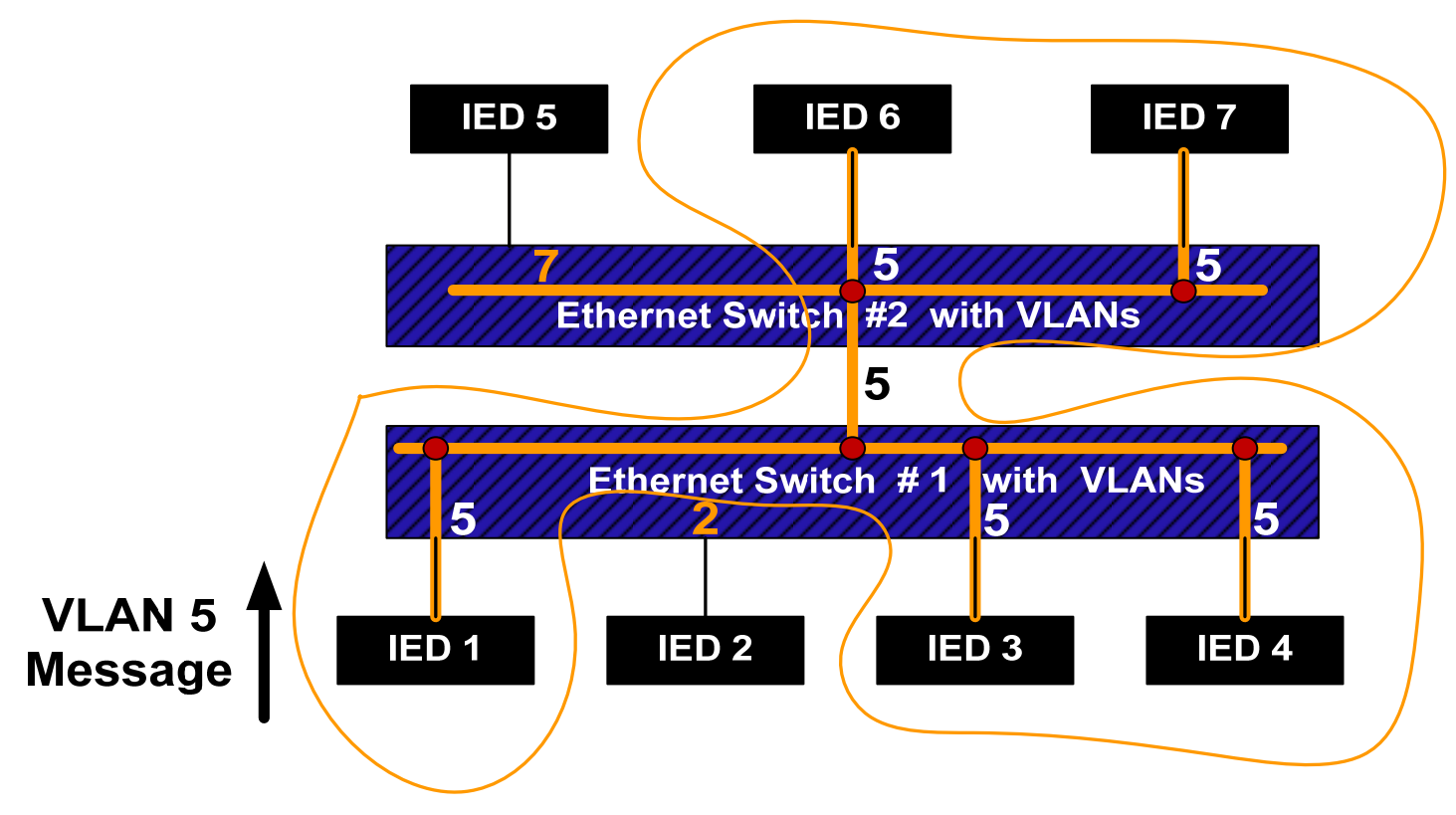

Figure 2.8 An Example of Network Traffic on VLAN No. 5

Each VLAN functions as a separate LAN. VLANs provide the capability of having multiple networks co-existing on the same Ethernet switch. The reason for creating multiple segments in Ethernet is to isolate broadcast domains. VLANs can isolate groups of users, or divide up traffic for security, bandwidth management, etc. A group of network users (ports) assigned to a VLAN form a broadcast domain. Data packets are forwarded only between ports that are designated for the same VLAN. Cross-domain broadcast traffic in the Ethernet switch is eliminated and bandwidth is saved by not allowing packets to flood all ports. For those reasons, a port may be configured to belong to multiple VLANs [14].

Interoperability

"Interoperability", as defined in IEC 61850 states that "Intelligent Electronic Devices (IEDs) from different manufacturers (have the) ability to operate on the same network or 
communication path sharing information and commands on a substation LAN" [15]. Having a common Substation Configuration Language (SCL), relays from different manufacturers can be setup to exchange data.

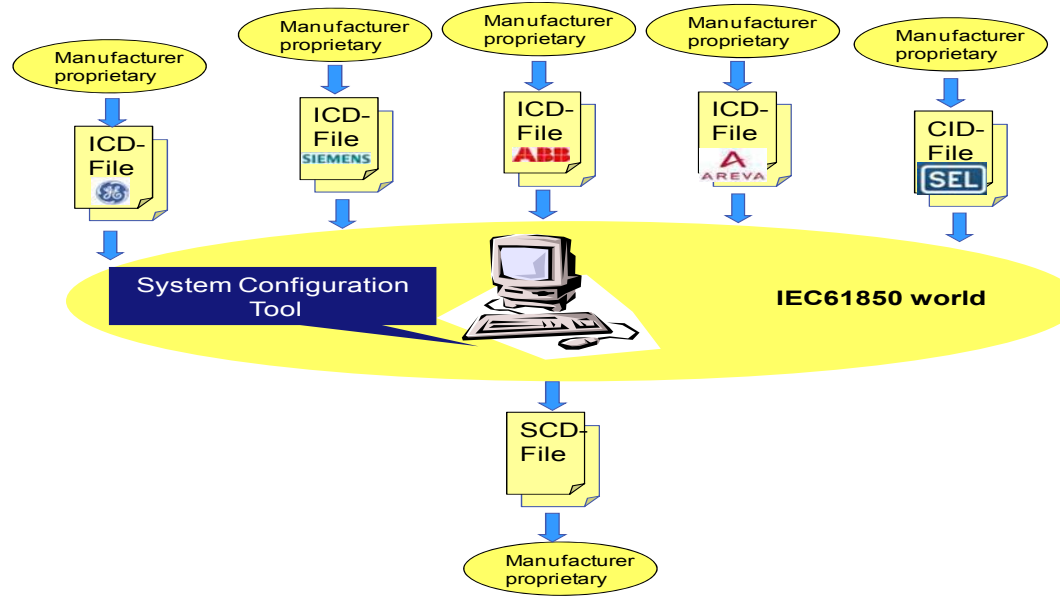

Figure 2.9 Configuration Tools for IEC 61850

As shown in Figure 2.9 [10], each relay manufacturer can create their own proprietary IED Capability Description (ICD) file. Each ICD file contains the IED logical nodes, data support, and services. By using a system configuration tool, the Substation Configuration Description (SCD) file can be created. The SCD contains all configured IEDs, the communication configuration and the complete station description.

For this study the interoperability of IEC 61850 is evaluated based on the complexity of how the relays were configured and the GOOSE blocking speed of each relay. 


\section{CHAPTER 3}

\section{PRIOR ART}

IEC 61850 Communications Protocol is an evolution of the electric utility communications protocol. This protocol is no longer a new face for the electric power utilities in Europe and Asia. In the US, however, because of the policies of cyber security and the stability requirements of the power grids, many electric power utilities have been reluctant to accept IEC 61850.

The general concept of implementing IEC 61850 on many protection schemes have been presented by many leading relay vendors [15] such as ABB [9, 13], GE Digital Energy Multilin $[6,10,14]$, and Schweitzer Engineering Laboratories (SEL). The implementation of fast bus protection scheme using IEC 61850 GOOSE messaging was reviewed in many papers referenced below.

The fast bus protection scheme was discussed briefly by Daqing Hou and Dave Dolezileck of SEL [1] to be one of the protection schemes that can be improved by using IEC 61850 standard.

Alex Apostolov published an article on the PACWorld magazine, " Impact of IEC 61850 on Bus Protection"'[2], which gave a short review of how multiple protective IEDs with IEC 61850 GOOSE messaging capability can be connected to the substation Local Area Network to perform the fast bus protection scheme. 
Veselin Skendzic and Armando Guzman of Schweitzer Engineering Laboratories published a paper titled, "Enhancing Power System Automation Through the Use of Real-Time Ethernet" [4], which illustrated the use of Generic Substation State Events (GSSE) (UCA 2.0 GOOSE) on the fast bus protection. GSSE (UCA 2.0 GOOSE) was an older and different version of IEC 61850 GOOSE.

Tony Zhao of Powell Electrical Systems, Inc., Lobomir Sevov and Craig Wester of GE Digital Energy Multilin, jointly published a paper, “Advanced Bus Transfer and Load Shedding Applications with IEC 61850" at the Texas A\&M 64th Relay Conference on April 13, 2011 [17]. The paper introduced different protection applications that can be implemented using IEC 61850.

"Status on the First IEC 61850 Based Protection and Control, Multi-Vendor Project in the United States" [18] was the first paper co-authored by the Tennessee Valley Authority and different relay vendors including GE Energy Digital Multilin, ABB, Siemens, and AREVA. This paper studied different protection schemes implementing IEC 61850 GOOSE messaging at a high voltage level of a $500-\mathrm{kV}$ substation.

There has not been a paper published by an independent end user using multi-vendor relays that studies the fast bus protection scheme, implementing IEC 61850 GOOSE messaging. For that reason, this study can be used by electric power utilities in determining if IEC 61850 is a viable replacement for the hardwired fast bus protection and other protection schemes as well. 


\section{CHAPTER 4}

\section{RELAY CONFIGURATIONS}

Fast Bus Protection Scheme Using the Traditional Hardwired Method

Fast bus protection for Feed 1 of Figure 2.1 was setup using two microprocessor-based relays. One was used for the feeder protection, and the other one was used for the bus protection. The schematic diagram of the fast bus protection scheme was configured as shown in Figure 4.1 below.
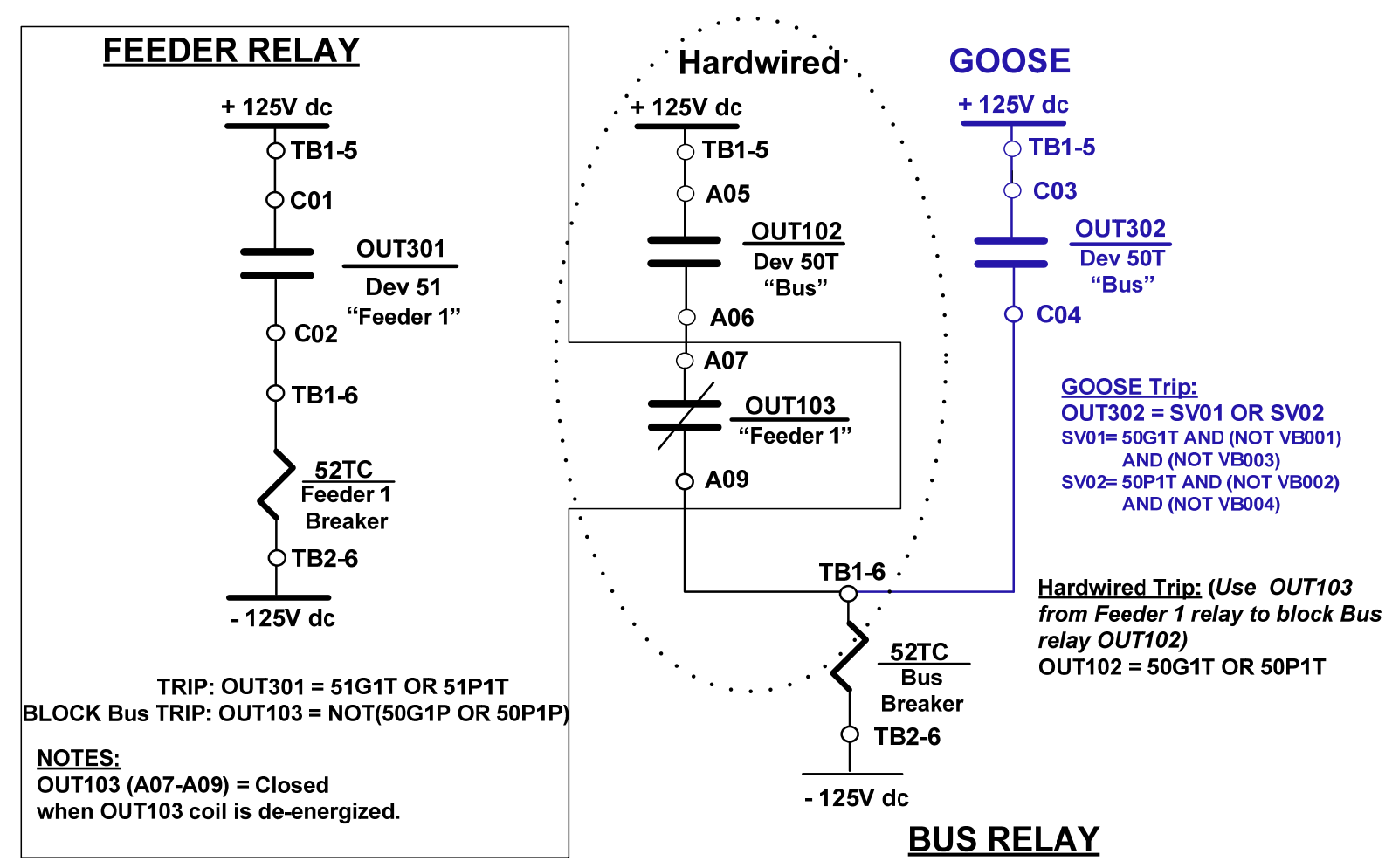

\section{BUS RELAY}

Fig 4.1 Fast Bus Protection Schemes for Feeder 1 
OUT103 is normally in the closed position, or OUT103 is equal to logic " 1 ". This means the $50 \mathrm{G} 1 \mathrm{P}$ or $50 \mathrm{P} 1 \mathrm{P}$ of the feeder relay is not picked up. In other words, there is no fault on the feeder. The logic is programmed as OUT103 $=$ NOT (50G1P OR 50P1P). 50G1P and 50P1P are the instantaneous overcurrent (IOC) residual ground and IOC phase elements in Device 50 of Feeder 1 relay.

$50 \mathrm{G} 1 \mathrm{P}$ or 50P1P of the feeder relay asserts instantaneously (without a setting time delay) if the feeder fault currents are above their pickup settings. The principle of the fast bus protection of microprocessor-based relays is the same as for EM relays. However, the bus relay's input contact coil (50B-1) that was used to toggle the bus relay output contact (50B-1) in the EM relay as shown in Figure 2.2 was eliminated here. OUT103 of the feeder relay replaces both input contact coil (50B-1) and output contact coil (50B-1) of the bus relay.

OUT102 of the bus relay as shown in the middle of Figure 4.1 was connected in series with the feeder relay's OUT103. OUT102 was programmed to trip for a bus fault through a short time delay $(\mathrm{T})$ of 3-4 cycles as OUT102 $=50 \mathrm{G} 1 \mathrm{~T}$ OR 50P1T. The 3-4 cycle time delay of the bus relay elements, 50G1T and 50P1T, were used to allow the feeder's relay output contact OUT103 to have enough time to open before the bus relay's output contact, OUT102, has time to close.

For a bus fault, Feeder 1 relay's output contact, OUT103, is already in the closed position. This is because the feeder relay will not see any current for a bus fault and element 50G1P or 50P1P of the feeder relay will not assert. The bus relay will trip and clear the fault as soon as its timer expires (either from 50G1T or 50P1T depending on the fault type).

The goal here was to measure the time that it took OUT103 of Feeder 1 relay where it went from the closed to open position starting from the point of the faults. This action was to 
measure the hardwired blocking speed that Feeder 1 relay takes to block the Bus relay from tripping for feeder faults.

Fast Bus Protection Scheme Using IEC 61850

An Ethernet switch and two microprocessor-based relays with IEC 61850 capabilities were used to set up a LAN for the fast bus protection scheme of Feeder 1 as shown in Figure 4.2. GOOSE messages sending from Feeder 1 relay and the virtual tripping input signals of the bus relay were used to inquire for feeder faults. The bus relay was set up as a subscriber while Feeder 1 relay was set up as the publisher.

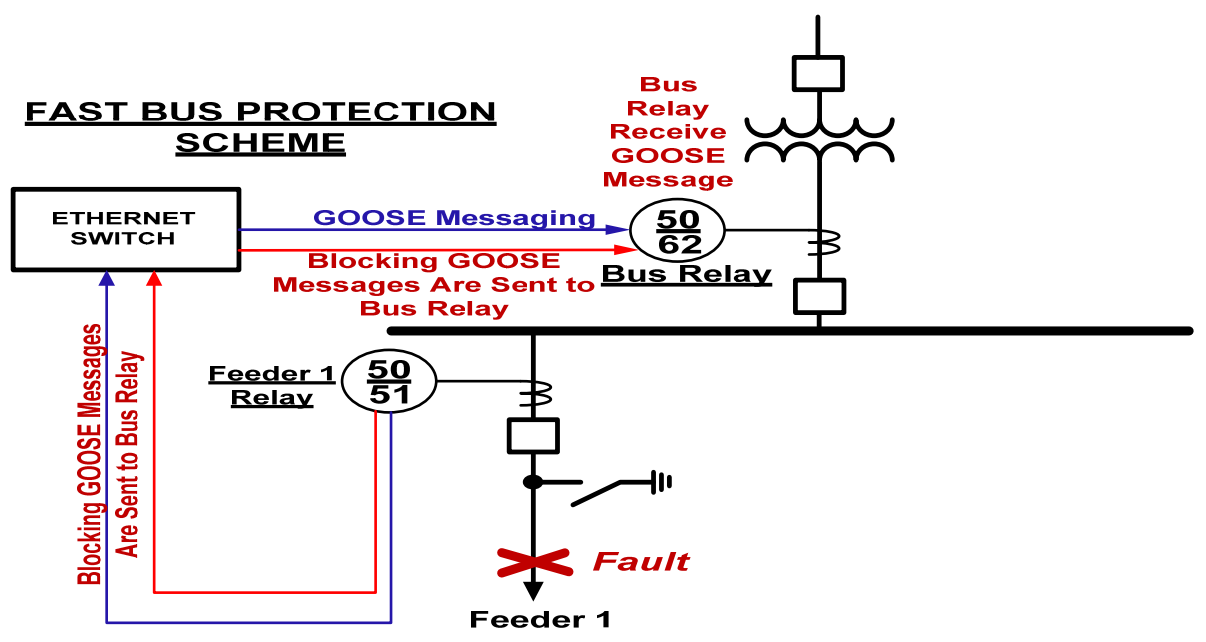

Fig 4.2 Fast Bus Protection Scheme for One Feeder Using IEC 61850

\section{Using Microprocessor-based Relays from Same Manufacture}

In this experiment, two microprocessor-based relays of the same relay manufacturer were used for Feeder 1 relay and the Bus relay. The goal for this part of the research was to measure the GOOSE blocking time that the Bus relay received from the Feeder 1 relay for the out of zone faults. The results of the experiment were then compared with the blocking time of the 
hardwired method. The main goal was to determine if the IEC 61850 method of the fast bus protection is a viable replacement for the hardwired fast bus protection method.

GOOSE Messages from the Publisher - Feeder 1 Relay (Vendor A)

The GOOSE messages for the IOC residual ground and phase elements from the publisher consisted of a unique data set as shown in Figure 4.3. These data sets follow the standard format of IEC 61850 that was illustrated previously as shown in Figure 2.5.
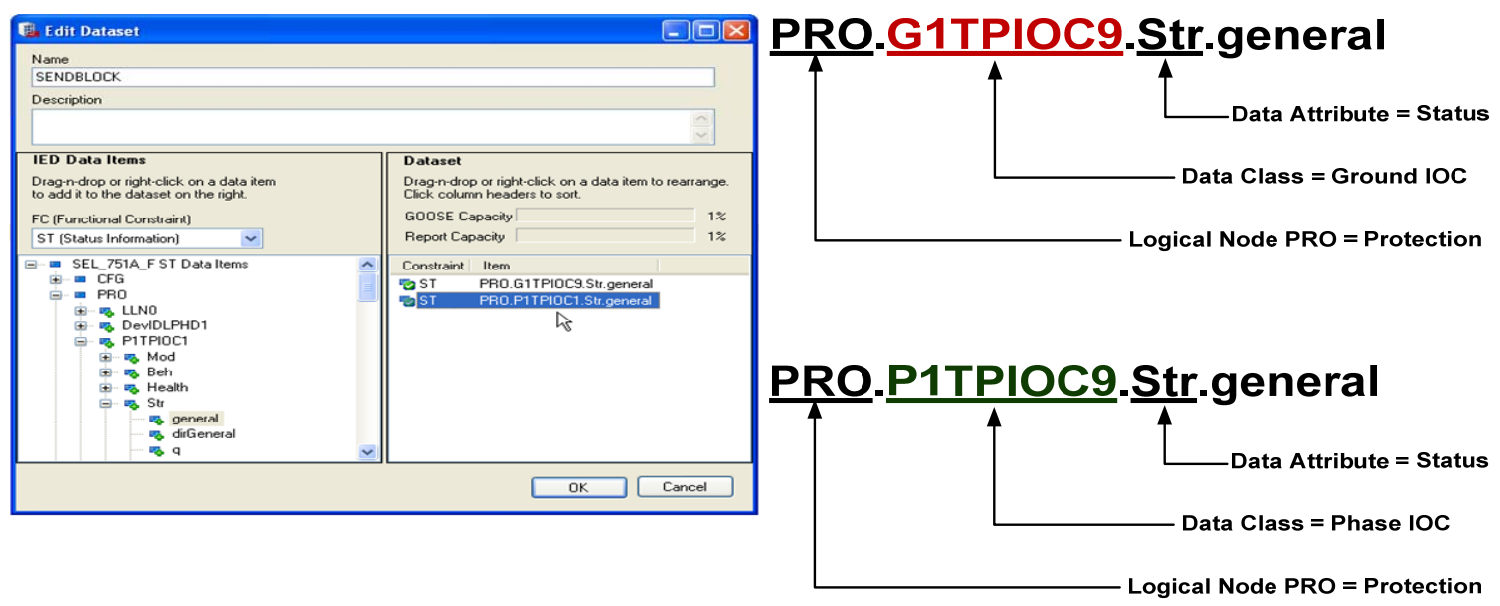

Fig 4.3 Data Set For Device 50 of Feeder 1's Relay

Each multicast GOOSE message has a unique GOOSE ID that was set up to communicate on VLAN No. 1 as shown in Figure 4.4. 


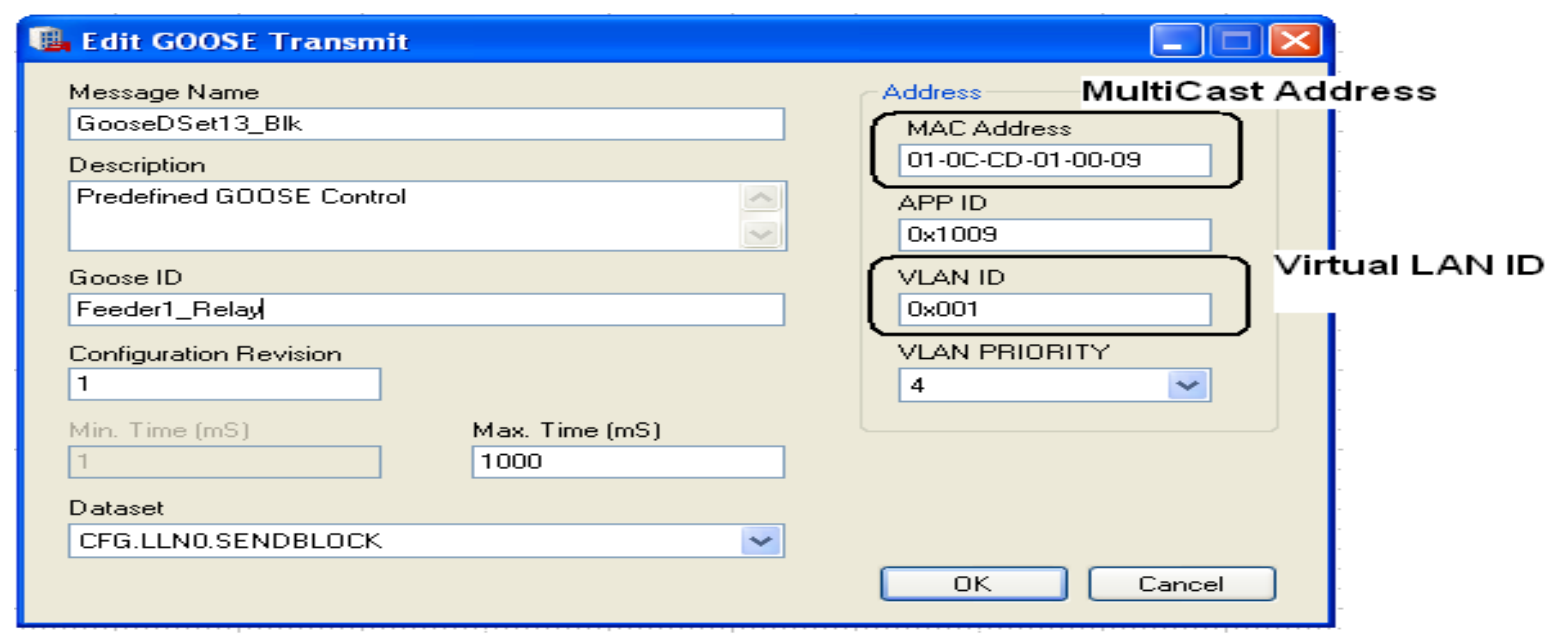

Fig 4.4 GOOSE Transmit Message for a Publisher

GOOSE Messages for the Subscriber - Bus Relay

Each GOOSE message for each relay element from the publisher (Feeder 1 relay) was mapped to a virtual control input of the subscriber (the bus relay). The process of using hard wires and relay inputs/outputs contacts to block the bus relay from operating for out of zone faults was replaced by a software mapping process as shown in Figure 4.5.

The GOOSE message for the IOC residual ground element of Feeder 1 relay was mapped into a virtual control input (VB001) of the bus relay while the IOC phase of the feeder relay was mapped into a virtual control input (VB002). OUT302 of the bus relay as shown in Figure 4.1 was programmed not to trip for an out of zone fault by detecting the insertion of VB001 or VB002 as follows:

$$
\begin{gathered}
\text { OUT302 = SV01 OR SV02, with } \\
\text { SV01 = 50G1T AND NOT (VB001), and } \\
\text { SV02 = 50P1PT AND NOT (VB002) }
\end{gathered}
$$




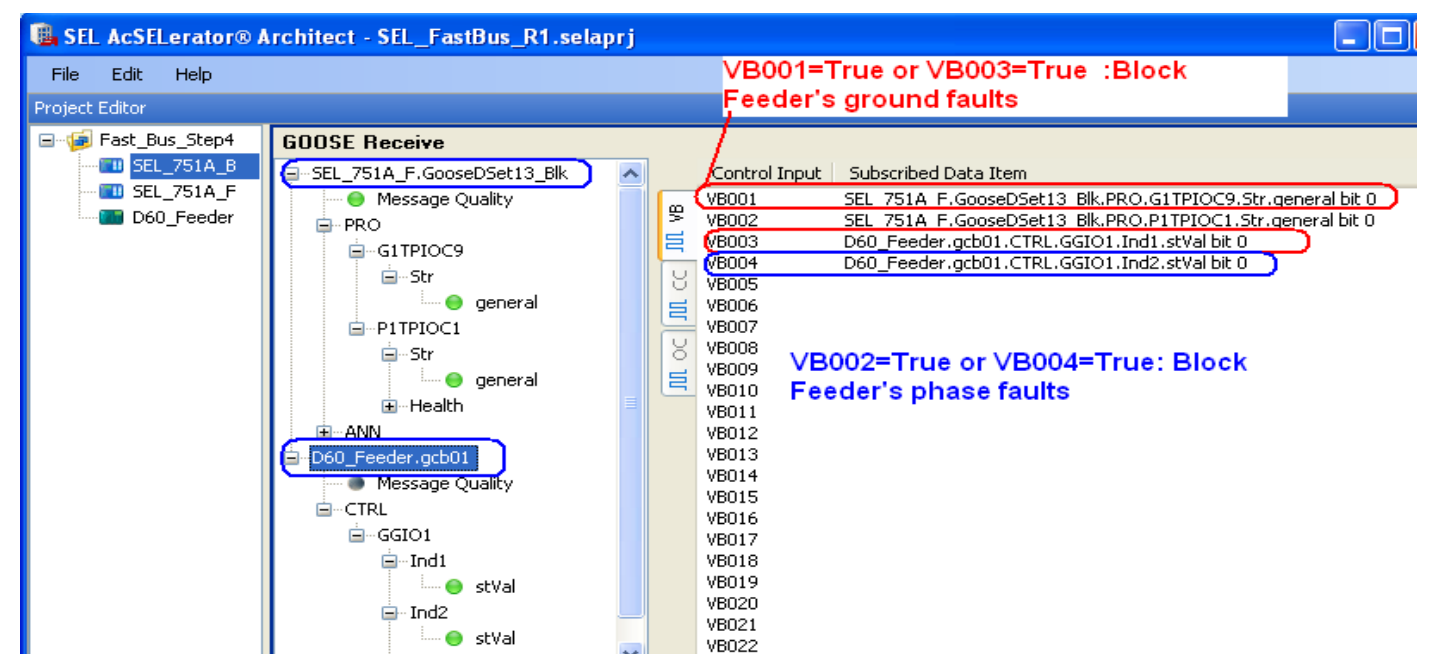

Figure 4.5 Mapping GOOSE Receive Messages

Using Microprocessor-based Relays from Different Manufacturers

In this part of the experiment, Feeder 1 relay was then replaced with a microprocessorbased relay from a different manufacturer. The purpose of this setup was to verify the interoperability of GOOSE messages between different relay manufacturers.

\section{GOOSE Messages from the Publisher - Feeder 1 Relay (Vendor B)}

Feeder 1 relay for this part of the experiment was made by a different relay manufacturer. All the GOOSE data set follow the same standard format of IEC 61850, but they are displayed uniquely. All GOOSE messages containing IEC 61850 data are collected in a dataset. The IOC residual ground element is called an IOC neutral ground element. The GOOSE messages for the IOC neutral and phase elements were assigned to the GOOSE transmission Dataset Item 1 (GGIO1.ST.Ind1.stVal) and Dataset Item 2 (GGIO1.ST.Ind2.stVal) as shown in Figure 4.6. As soon as the status of these relay elements change, the GOOSE transmission data transmit the GOOSE messages to block the bus relay from tripping for the feeder faults. 


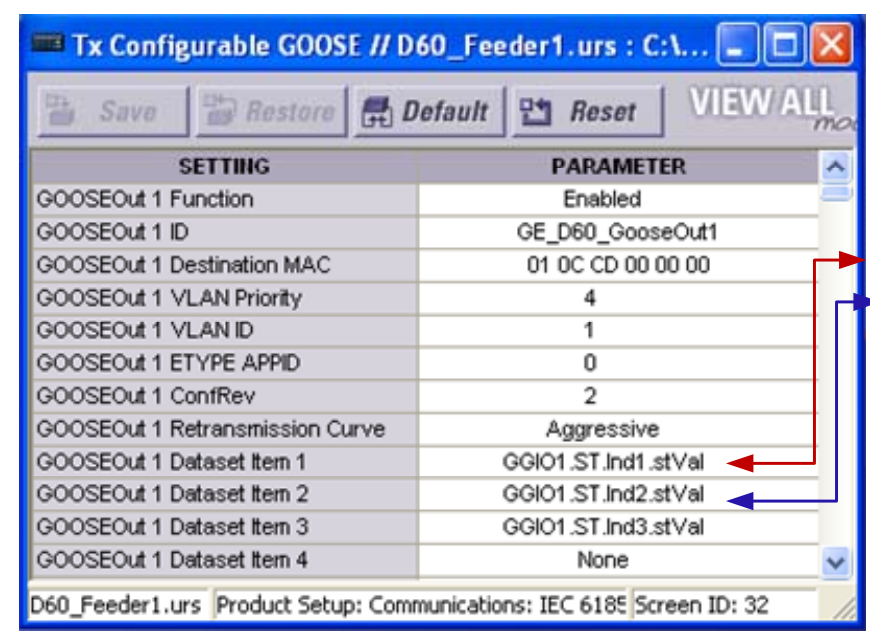

Figure 4.6 Mapping GOOSE Transmit Messages for a Publisher

\begin{tabular}{|l|c|c|}
\hline GGI01 Status Configuration II D60_Feed... \\
\hline SETTIIIG
\end{tabular}

The GOOSE messages for the IOC neutral ground of Feeder 1 relay were mapped into the virtual control input VB003 of the Bus relay, while the IOC phase of Feeder 1 relay were mapped into the virtual control input VB004.

The bus relay was finally set up as a subscriber for Feeder 1 with the existence of both relays acting as the Feeder 1 relay from different manufacturers. This set up is normally done when one relay is served as a primary protection while the second relay is served as a backup or redundancy protection. The bus relay output contact was programmed by adding the conditions of AND NOT (VB003) to the original SV01 equation for ground fault detection while adding the conditions of AND NOT (VB004) for phase faults as follows:

$$
\text { OUT302 = SV01 OR SV02 }
$$

$$
\mathrm{SV01}=50 \mathrm{G} 1 \mathrm{~T} \text { AND NOT (VB001) AND NOT (VB003) }
$$$$
\mathrm{SV02}=50 \mathrm{G} 1 \mathrm{~T} \text { AND NOT (VB002) AND NOT (VB004) }
$$ 
The bus relay was programmed not to trip for an out of zone fault by detecting the insertion of VB001 and VB003 for ground faults or VB002 and VB004 for phase faults for both relays from Vendor A and Vendor B as shown in Figure 4.5. 


\section{CHAPTER 5}

\section{TEST PROCEDURES AND RESULTS}

\section{Test Procedures}

The laboratory setup of this experiment is shown in Figure 5.1. Feeder 1 relay was mounted on the upper left of the relay test rack while the bus relay was mounted on the upper right of the relay test rack. These two relays are made from the same relay manufacture. Feeder 2 relay is a relay made from a different relay manufacture. It is mounted right below the RuggedCom Ethernet test switch. Three breaker simulators were used to simulate Feeder 1 breaker, Feeder 2 breaker, and Bus breaker labeled as F1, F2, and Bus accordingly. The relay test switches were also used to support the testing purpose.

An Omicron CMC 256-6 test set was used to supply six current sources. Three current test leads (A, B, and C phase) were used to inject phase currents $\mathrm{A}, \mathrm{B}$, and $\mathrm{C}$ to the feeder relay. The other three current test leads were applied to the bus relay accordingly. The feeder relay and the bus relay received the same simulated fault currents from the same test set simultaneously, therefore, no time synchronizing equipment was needed. The setting for Device 51 of Feeder 1 relay was omitted in this study because this element operates independently regarding the fast bus protection scheme. 


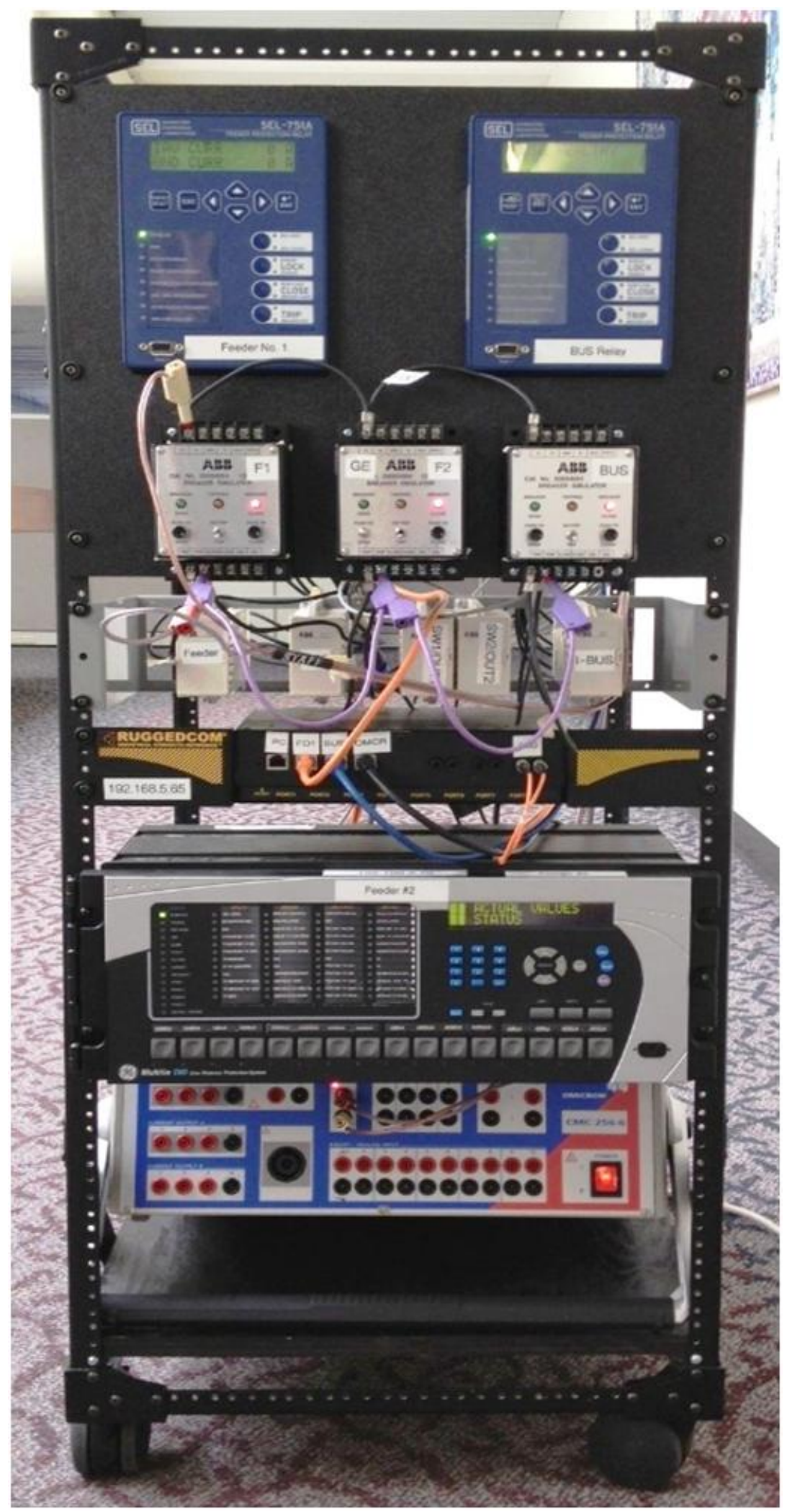

Figure 5.1 An Image of the Laboratory Setup for The Study 
This research was divided into three experiments: the Hardwired Method, the IEC 61850 GOOSE Messaging Method Using the Same Relay Manufacture, and the IEC 61850 GOOSE Messaging Method Using Different Relay Manufactures.

Two separate sets of relay settings were applied to the feeder relay and the bus relay each time, for each individual experiment. Two types of feeder faults were used: A phase-to-Ground fault and A phase-to-B phase fault. The fault current magnitudes of $12.5 \mathrm{~A}, 10.75 \mathrm{~A}, 9.5 \mathrm{~A}, 8.5 \mathrm{~A}$, and 5.0A for each fault type were applied to the feeder relay and the bus relay simultaneously for each set of relay settings in each experiment. The purpose of this setup was to observe if there would be any impact on the blocking speeds of the feeder relay if the ratios of fault currents to the pickup settings of the feeder relay were different.

All the test results were recorded and grouped under different pickup settings and different fault types for the feeder relay. They were displayed as shown in Table 5.1-5.4.

The Hardwired Method

This experiment measured the time that the feeder relay's output contact (OUT103) took to go from closed to open starting from fault inception. The main objective here was to evaluate how fast the feeder relay's output contact could block the bus relay from operating for the out of zone faults. Different magnitudes of fault currents (12.5A, 10.75A, 9.5A, 8.5A, and 5.0A) were applied the feeder relay and the bus relay using two different sets of settings as described below. 


\section{Testing Using High Ratios of Multiples of Pickup of Settings}

The feeder relay's IOC residual ground and phase element were set to $0.75 \mathrm{~A}$ and $2.0 \mathrm{~A}$, respectively. The bus relay's IOC residual ground and phase element were set to $1.0 \mathrm{~A}$ and $2.25 \mathrm{~A}$, respectively. The maximum load currents were assumed to be $1.0 \mathrm{~A}$.

\section{Testing Using Low Ratios of Multiples of Pickup of Settings}

The feeder relay's IOC residual ground and phase element were then set to $4.0 \mathrm{~A}$ and 8.0A, respectively. The bus relay's IOC residual ground and phase element were set to $4.25 \mathrm{~A}$ and $8.25 \mathrm{~A}$, respectively. The maximum load currents were assumed to be $2.0 \mathrm{~A}$.

IEC 61850 GOOSE Messaging Method Using the Same Relay Manufacturer

This experiment measured the GOOSE blocking time that the bus relay received from the feeder relay for the out of zone faults. Two microprocessor-based relays of the same relay manufacturer were used. This method will be referred to as the IEC 61850 same manufacturer relay method. The same test setups for the hardwired method were repeated here.

The bus relay virtual inputs' VB001 and VB002 were set up to be asserted when GOOSE messages were received for a feeder's ground fault and a feeder's phase fault respectively. Because the Omicron test set can only measure the actual GOOSE transmitting time but not the actual GOOSE receiving time starting from the point of fault, VB001 and VB002 were mapped to two independent physical output contacts (OUT303 and OUT304) respectively. The actual GOOSE receiving time for each fault was then calculated by subtracting the relay output contact closing time of $4.0 \mathrm{~ms}[19,20]$ from the operating time for OUT303 or OUT304 starting from fault inception. 
IEC 61850 GOOSE Messaging Method Using Different Relay Manufacturers

This experiment measured the GOOSE blocking time that the bus relay received from the feeder relay for the out of zone faults. Two microprocessor-based relays of different relay manufacturers were used. This method will be referred to as the IEC 61850 mixed manufacturer relay method. The same test setup for Part 1 was repeated. The bus relay virtual inputs' VB003 and VB004 were set up to be asserted when GOOSE messages were received for a feeder's ground fault and a feeder's phase fault respectively. VB003 and VB004 were mapped to OUT303 and OUT304, respectively.

Test Results

Table 5.1

Ground Faults’ Blocking Speeds Using High Ratios of Multiples of Pickup Settings

\begin{tabular}{|c|c|c|c|c|c|c|c|c|c|c|c|c|c|c|c|}
\hline \multicolumn{16}{|c|}{$\begin{array}{c}\text { Feeder Relay: } \mathrm{RIOC} / \mathrm{NIOC}=0.75 \mathrm{~A}, \mathrm{PIOC}=2.0 \mathrm{~A} \\
\text { Bus Relay: } \mathrm{RIOC} / \mathrm{NIOC}=1.0 \mathrm{~A}, \mathrm{PIOC}=2.25 \mathrm{~A}\end{array}$} \\
\hline $\begin{array}{l}\text { Feeder's Ground Faults in } \\
\text { amperes }\end{array}$ & \multicolumn{3}{|c|}{12.50} & \multicolumn{3}{|c|}{10.75} & \multicolumn{3}{|c|}{9.50} & \multicolumn{3}{|c|}{8.50} & \multicolumn{3}{|c|}{5.00} \\
\hline Calculated & \multicolumn{3}{|c|}{16.67} & \multicolumn{3}{|c|}{14.33} & \multicolumn{3}{|c|}{12.67} & \multicolumn{3}{|c|}{11.33} & \multicolumn{3}{|c|}{6.67} \\
\hline $\begin{array}{l}\text { Feeder Relay and Bus Relay } \\
\text { use: } \\
==========\text { ’〉》 }\end{array}$ & $\begin{array}{c}\text { Diff } \\
\text { Vendors }\end{array}$ & \begin{tabular}{|c|} 
Same \\
Vendor
\end{tabular} & Hardwired & $\mid$\begin{tabular}{c|} 
Diff \\
Vendors
\end{tabular} & \begin{tabular}{|c|} 
Same \\
Vendor
\end{tabular} & Hardwired & $\mid \begin{array}{c}\text { Diff } \\
\text { Vendors }\end{array}$ & \begin{tabular}{|l} 
Same \\
Vendor
\end{tabular} & Hardwired & $\begin{array}{c}\text { Diff } \\
\text { Vendors }\end{array}$ & \begin{tabular}{|c|} 
Same \\
Vendor
\end{tabular} & Hardwired & $\begin{array}{c}\text { Diff } \\
\text { Vendors }\end{array}$ & \begin{tabular}{|c|} 
Same \\
Vendor
\end{tabular} & Hardwired \\
\hline $\begin{array}{l}\text { Feeder Relay's GOOSE } \\
\text { Starting time (in ms) }\end{array}$ & 8.00 & 6.00 & & 10.90 & 6.10 & & 11.40 & 5.40 & & 11.60 & 3.90 & & 13.10 & 5.60 & \\
\hline $\begin{array}{l}\text { Bus Relay's GOOSE Received } \\
\text { Time (in ms), including a } 4 \mathrm{~ms} \\
\text { of relay contact closing time }\end{array}$ & 14.00 & 11.20 & & 15.50 & 12.20 & & 19.10 & 10.40 & & 17.20 & 9.90 & & 20.10 & 11.10 & \\
\hline $\begin{array}{l}\text { OUT103 going from Close to } \\
\text { Open (in ms) }\end{array}$ & & & 9.50 & & & 9.40 & & & 8.90 & & & 7.40 & & & 9.00 \\
\hline $\begin{array}{l}\text { Bus Trip (in ms) for Bus } \\
\text { Faults (not Feeder 1's Faults) }\end{array}$ & \multicolumn{3}{|c|}{59.80} & \multicolumn{3}{|c|}{61.90} & \multicolumn{3}{|c|}{67.80} & \multicolumn{3}{|c|}{62.60} & \multicolumn{3}{|c|}{68.90} \\
\hline
\end{tabular}


Table 5.2

Phase Faults' Blocking Speeds Using High Ratios of Multiples of Pickup Settings

\begin{tabular}{|c|c|c|c|c|c|c|c|c|c|c|c|c|c|c|c|}
\hline \multicolumn{16}{|c|}{$\begin{array}{c}\text { Feeder Relay: } \mathrm{RIOC} / \mathrm{NIOC}=0.75 \mathrm{~A}, \mathrm{PIOC}=2.0 \mathrm{~A} \\
\text { Bus Relay: } \mathrm{RIOC} / \mathrm{NIOC}=1.0 \mathrm{~A}, \mathrm{PIOC}=2.25 \mathrm{~A}\end{array}$} \\
\hline $\begin{array}{l}\text { Feeder's Phase Faults } \\
\text { in amperes }\end{array}$ & \multicolumn{3}{|c|}{12.50} & \multicolumn{3}{|c|}{10.75} & \multicolumn{3}{|c|}{9.50} & \multicolumn{3}{|c|}{8.50} & \multicolumn{3}{|c|}{5.00} \\
\hline $\begin{array}{l}\text { Calculated Multiple of Pickup } \\
\text { for Feeder } 1 \text { Relay }\end{array}$ & \multicolumn{3}{|c|}{6.25} & \multicolumn{3}{|c|}{5.38} & \multicolumn{3}{|c|}{4.75} & \multicolumn{3}{|c|}{4.25} & \multicolumn{3}{|c|}{2.50} \\
\hline $\begin{array}{l}\text { Feeder Relay and Bus Relay } \\
\text { use: } \\
===========\gg \gg \gg\end{array}$ & $\begin{array}{c}\text { Diff } \\
\text { Vendors }\end{array}$ & $\begin{array}{c}\text { Same } \\
\text { Vendor }\end{array}$ & Hardwired & $\begin{array}{c}\text { Diff } \\
\text { Vendors }\end{array}$ & $\begin{array}{c}\text { Same } \\
\text { Vendor }\end{array}$ & Hardwired & $\begin{array}{c}\text { Diff } \\
\text { Vendors }\end{array}$ & $\begin{array}{c}\text { Same } \\
\text { Vendor }\end{array}$ & Hardwired & $\begin{array}{c}\text { Diff } \\
\text { Vendors }\end{array}$ & $\begin{array}{c}\text { Same } \\
\text { Vendor }\end{array}$ & Hardwired & $\begin{array}{c}\text { Diff } \\
\text { Vendors }\end{array}$ & $\begin{array}{c}\text { Same } \\
\text { Vendor }\end{array}$ & Hardwired \\
\hline $\begin{array}{l}\text { Feeder Relay's GOOSE } \\
\text { Starting time (in ms) }\end{array}$ & 7.20 & 4.40 & & 7.60 & 11.40 & & 8.10 & 8.90 & & 8.60 & 10.70 & & 12.50 & 11.90 & \\
\hline $\begin{array}{l}\text { Bus Relay's GOOSE Received } \\
\text { Time (in ms), including a } 4 \mathrm{~ms} \\
\text { of relay contact closing time }\end{array}$ & 18.20 & 11.60 & & 13.60 & 18.50 & & 11.80 & 16.10 & & 12.00 & 17.90 & & 18.50 & 15.90 & \\
\hline $\begin{array}{l}\text { OUT103 going from Close to } \\
\text { Open (in ms) }\end{array}$ & & & 7.80 & & & 14.90 & & & 12.30 & 13.60 & & 14.10 & & & 15.40 \\
\hline $\begin{array}{l}\text { Bus Trip (in ms) for Bus } \\
\text { Faults (not Feeder 1's Faults) }\end{array}$ & \multicolumn{3}{|c|}{65.90} & \multicolumn{3}{|c|}{66.60} & \multicolumn{3}{|c|}{66.60} & \multicolumn{3}{|c|}{69.00} & \multicolumn{3}{|c|}{73.80} \\
\hline
\end{tabular}

Table 5.3

Ground Faults' Blocking Speeds Using Low Ratios of Multiples of Pickup Settings

\begin{tabular}{|c|c|c|c|c|c|c|c|c|c|c|c|c|c|c|c|}
\hline \multicolumn{16}{|c|}{$\begin{array}{l}\text { Feeder Relay: } \mathrm{RIOC} / \mathrm{NIOC}=4.0 \mathrm{~A}, \mathrm{PIOC}=8.0 \mathrm{~A} \\
\text { Bus Relay: } \mathrm{RIOC} / \mathrm{NIOC}=4.25 \mathrm{~A}, \mathrm{PIOC}=8.25 \mathrm{~A}\end{array}$} \\
\hline $\begin{array}{l}\text { Feeder's Ground Faults } \\
\text { in amperes }\end{array}$ & \multicolumn{3}{|c|}{12.50} & \multicolumn{3}{|c|}{10.75} & \multicolumn{3}{|c|}{9.50} & \multicolumn{3}{|c|}{8.50} & \multicolumn{3}{|c|}{5.00} \\
\hline $\begin{array}{l}\text { Calculated } \\
\text { Multiple Of Pickup for Feeder } \\
1 \text { Relay }\end{array}$ & \multicolumn{3}{|c|}{3.13} & \multicolumn{3}{|c|}{2.69} & \multicolumn{3}{|c|}{2.38} & \multicolumn{3}{|c|}{2.13} & \multicolumn{3}{|c|}{1.25} \\
\hline $\begin{array}{l}\text { Feeder Relay and Bus Relay } \\
\text { use: } \\
============\gg \gg 〉\end{array}$ & $\begin{array}{c}\text { Diff } \\
\text { Vendors }\end{array}$ & $\begin{array}{c}\text { Same } \\
\text { Vendor }\end{array}$ & Hardwired & $\begin{array}{c}\text { Diff } \\
\text { Vendors }\end{array}$ & $\begin{array}{c}\text { Same } \\
\text { Vendor }\end{array}$ & Hardwired & $\begin{array}{c}\text { Diff } \\
\text { Vendors }\end{array}$ & $\begin{array}{c}\text { Same } \\
\text { Vendor }\end{array}$ & Hardwired & $\begin{array}{c}\text { Diff } \\
\text { Vendors }\end{array}$ & $\begin{array}{c}\text { Same } \\
\text { Vendor }\end{array}$ & Hardwired & $\begin{array}{c}\text { Diff } \\
\text { Vendors }\end{array}$ & $\begin{array}{c}\text { Same } \\
\text { Vendor }\end{array}$ & Hardwired \\
\hline $\begin{array}{l}\text { Feeder Relay's GOOSE Starting } \\
\text { time (in ms) }\end{array}$ & 17.50 & 9.90 & & 17.20 & 10.80 & & 18.00 & 14.00 & & 18.10 & 17.70 & & 28.30 & & \\
\hline $\begin{array}{l}\text { Bus Relay's GOOSE Received } \\
\text { Time (in ms), including a } 4 \mathrm{~ms} \\
\text { of relay contact closing time }\end{array}$ & 21.70 & 13.50 & & 23.80 & 14.90 & & 22.50 & 18.80 & & 24.90 & 21.70 & & 33.00 & & \\
\hline $\begin{array}{l}\text { OUT103 going from Close to } \\
\text { Open (in ms) }\end{array}$ & & & 13.30 & & & 14.00 & & & 17.50 & & & 21.20 & & & 21.00 \\
\hline \begin{tabular}{|l} 
Bus Trip (in ms) for Bus Faults \\
(not Feeder 1's Faults)
\end{tabular} & & 66.80 & & & 67.20 & & & 68.60 & & & 75.40 & & & 81.40 & \\
\hline
\end{tabular}


Table 5.4

Phase Faults' Blocking Speeds Using Low Ratios of Multiples of Pickup Settings

\begin{tabular}{|c|c|c|c|c|c|c|c|c|c|c|c|c|c|c|c|}
\hline \multicolumn{16}{|c|}{$\begin{array}{l}\text { Feeder Relay: } \mathrm{RIOC} / \mathrm{NIOC}=4.0 \mathrm{~A}, \mathrm{PIOC}=8.0 \mathrm{~A} \\
\text { Bus Relay: } \mathrm{RIOC} / \mathrm{NIOC}=4.25 \mathrm{~A}, \mathrm{PIOC}=8.25 \mathrm{~A}\end{array}$} \\
\hline $\begin{array}{l}\text { Feeder's Phase Faults } \\
\text { in amperes }\end{array}$ & \multicolumn{3}{|c|}{12.50} & \multicolumn{3}{|c|}{10.75} & \multicolumn{3}{|c|}{9.50} & \multicolumn{3}{|c|}{8.50} & \multicolumn{3}{|c|}{5.00} \\
\hline $\begin{array}{l}\text { Calculated } \\
\text { Multiple Of Pickup for Feeder } \\
1 \text { Relay }\end{array}$ & \multicolumn{3}{|c|}{1.56} & \multicolumn{3}{|c|}{1.34} & \multicolumn{3}{|c|}{1.19} & \multicolumn{3}{|c|}{1.06} & \multicolumn{3}{|c|}{0.63} \\
\hline $\begin{array}{l}\text { Feeder Relay and Bus Relay } \\
\text { use: } \\
\text { ===========-〉〉〉 }\end{array}$ & $\begin{array}{c}\text { Diff } \\
\text { Vendors }\end{array}$ & $\begin{array}{c}\text { Same } \\
\text { Vendor }\end{array}$ & Hardwired & $\begin{array}{c}\text { Diff } \\
\text { Vendors }\end{array}$ & $\begin{array}{c}\text { Same } \\
\text { Vendor }\end{array}$ & Hardwired & $\begin{array}{c}\text { Diff } \\
\text { Vendors }\end{array}$ & $\begin{array}{c}\text { Same } \\
\text { Vendor }\end{array}$ & Hardwired & $\mid \begin{array}{c}\text { Diff } \\
\text { Vendors }\end{array}$ & $\begin{array}{c}\text { Same } \\
\text { Vendor }\end{array}$ & Hardwired & $\begin{array}{c}\text { Diff } \\
\text { Vendors }\end{array}$ & $\begin{array}{c}\text { Same } \\
\text { Vendor }\end{array}$ & Hardwired \\
\hline $\begin{array}{l}\text { Feeder Relay's GOOSE Starting } \\
\text { time (in } \mathrm{ms} \text { ) }\end{array}$ & 15.90 & 17.50 & & 19.10 & 20.40 & & 21.50 & 19.60 & & 22.40 & 24.30 & & & No Trip & \\
\hline $\begin{array}{l}\text { Bus Relay's GOOSE Received } \\
\text { Time (in ms), including a } 4 \mathrm{~ms} \\
\text { of relay contact closing time }\end{array}$ & 21.30 & 22.60 & & 24.90 & 25.70 & & & 24.90 & & 27.50 & 28.60 & & & No Trip & \\
\hline $\begin{array}{l}\text { OUT103 going from Close to } \\
\text { Open (in ms) }\end{array}$ & & & 20.90 & & & 23.90 & 26.10 & & 23.00 & & & 27.70 & & & No Trip \\
\hline $\begin{array}{l}\text { Bus Trip (in ms) for Bus Faults } \\
\text { (not Feeder 1's Faults) }\end{array}$ & & 75.70 & & & 75.60 & & & 82.20 & & & No Tri & & & No Trip & \\
\hline
\end{tabular}




\section{CHAPTER 6}

\section{DISCUSSIONS}

The goal of this research was to evaluate if IEC 61850 was an optimal solution for the fast bus protection scheme for the radial distribution systems. The evaluation was done by comparing the IEC 61850 GOOSE messaging method to the traditional hardwired method. The comparison was based on the criteria of the system disturbance clearing time, the engineering cost and construction cost, and the interoperability of this new protocol between relays of different manufacturers. IEC 61850 would not be considered as an optimal solution for the traditional hardwired fast bus protection scheme if any of the criteria was not met.

System Disturbance Clearing Time

\section{Average Time Latency of GOOSE Messages}

One of the main focuses in this research was to measure the average time latency that a GOOSE message could detect a change in the analog measurements or in other words how fast a GOOSE message could detect a fault. This study verifies that a well-designed IED can perform a detection of change with an average latency of $1 \mathrm{~ms}$ in the receiving device [6] as follows.

For the fast bus protection scheme, GOOSE messages detect a feeder fault condition when the IOC measurements are higher than the feeder relay's pickup values (or the feeder relay's preset pickup settings). The IOC for the phase and ground elements (Device 50) by definition of the American National Standards Institute (ANSI) has no time delay. It means no 
external setting time delay. These elements actually have different internal time delay pickups based on the different ratios of the applied currents to the relay's pickup settings. These ratios are called the multiples of pickup settings for the IOC elements. Each relay manufacturer has different specifications. However, the specifications of each relay manufacturer are not so different from each other. This is because most of the relay manufacturers in the U.S. follow the ANSI standards.

The time latency of GOOSE detection for a feeder fault is the difference of the measured GOOSE starting time and the IOC's internal time delay pickup, starting from fault inception. The interpretation of GOOSE time latency is illustrated as shown in Figure 6.1 by using one of the test data of Table 5.1.

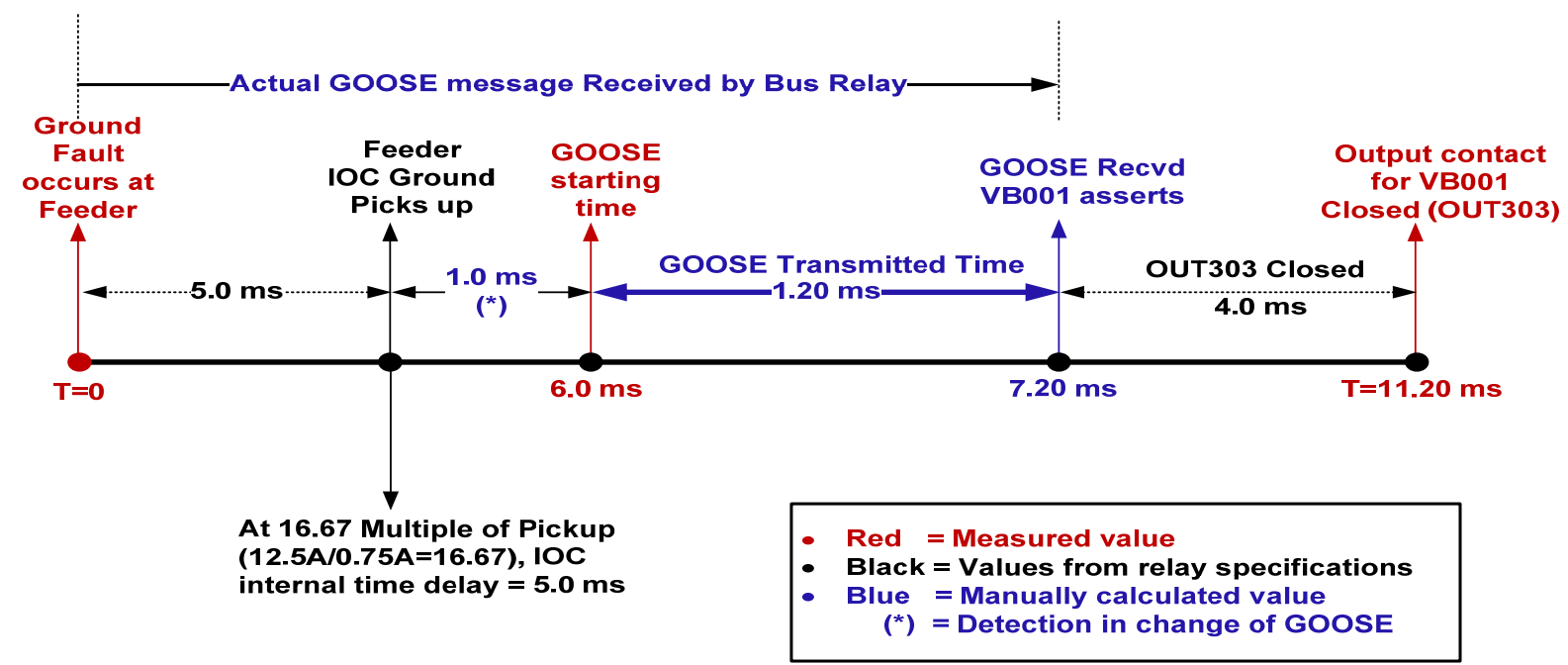

Figure 6.1 GOOSE Capturing Time Using the Omicron Test Set and Relay Specifications

The residual ground IOC (RIOC) of Feeder 1 relay, as shown in Figure 6.1, was set to pickup at $0.75 \mathrm{~A}$. The RIOC is also referred to as the neutral ground IOC (NIOC) throughout this study. 
Applying an A phase-to-Ground fault current of $12.5 \mathrm{~A}$, or at 16.67 multiple of the pickup setting $(12.5 \mathrm{~A} / 0.75 \mathrm{~A}=16.67)$, requires the RIOC from $3.67 \mathrm{~ms}(0.22$ cycles $)$ to $8.30 \mathrm{~ms}$ (0.50cycles) to assert per relay specifications [19]. Assuming that it took the NIOC $5.0 \mathrm{~ms}$ to pickup, and the feeder relay's GOOSE starting time was measured to be $6.0 \mathrm{~ms}$, as shown in Table 5.1, then it would take the GOOSE message approximate $1.0 \mathrm{~ms}$ to detect the fault as illustrated in Figure 6.1.

The average time latency of GOOSE detection is approximately $1.0 \mathrm{~ms}$ for the multiples of relay pickup settings that are greater than 5.0. The average latency is slightly slower than 1.0 ms for the multiples of relay pickup settings that are smaller than 4.0. This experimental data proves a well designed IED can perform a detection of change with an average latency of $1 \mathrm{~ms}$ in the receiving device.

\section{Bus Relay's Blocking Time}

The goal of this research was to determine if the implementation of IEC 61850 could improve the system disturbance clearing time per references $[1,2,6,11,21]$. The blocking time that the bus relay received from the feeder relay using the hardwired method and the IEC 61850 methods was captured and compared. The faster speed or the shorter time that the feeder relay can block the bus relay, the shorter time the bus relay can be set to clear their in-zone faults.

Figure 6.2 is a graphical display of the test results of Table 5.1 and Table 5.3 for ground faults on Feeder 1. Note that $4.0 \mathrm{~ms}$ (relay's output contact operating time) was deducted for the two IEC 61850 methods but not the hardwired method. Figure 6.2 shows that the hardwired method had a faster blocking speed compared to the IEC 61850 mixed manufacturer relay method. However, it had a slower blocking speed compared to the IEC 61850 same 
manufacturer relay method. Although the display of Figure 6.2 shows that the hardwired method had a much better blocking performance compared to the IEC 61850 mixed manufacturer relay method, the maximum time difference was only $7.0 \mathrm{~ms}$ which is insignificant. The results of the ground faults of Feeder 1 show that the IEC 61850 GOOSE messaging method did not always trip faster compared to the hardwired method.

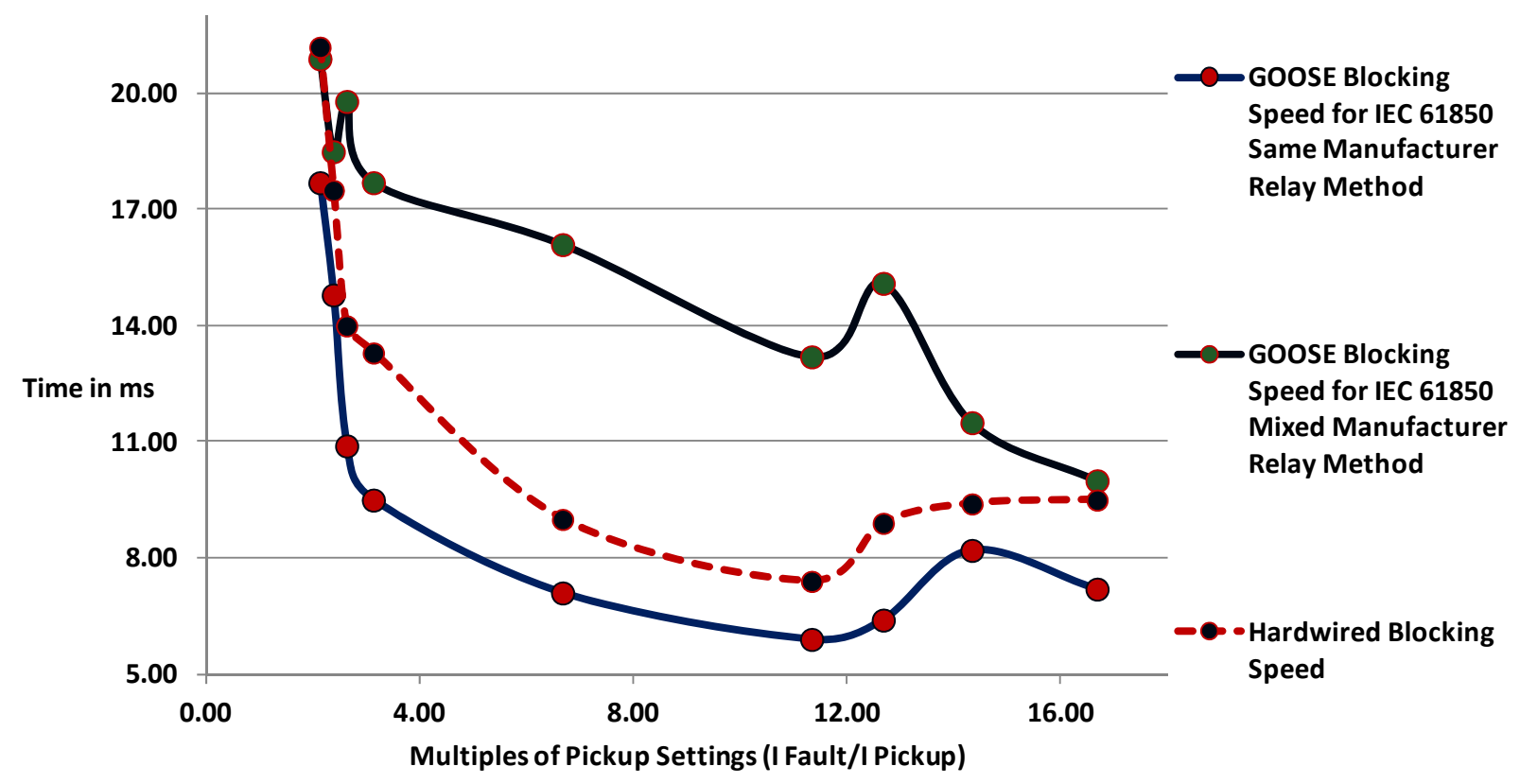

Figure 6.2 The Actual GOOSE Blocking Speed Versus Hardwired Speed for Ground Faults 
The test results of Table 5.2 and Table 5.4 were used to graph the blocking speed of the feeder relay for the out of zone phase faults for all three methods as shown in Figure 6.3. By observing the graph within the range of 4.25 to 5.75 for the multiples of pickup settings, the hardwired method and the IEC 61850 mixed manufacturer relay method had the same blocking speed. These two methods, however, were both slower than the IEC 61850 mixed manufacturer relay method.

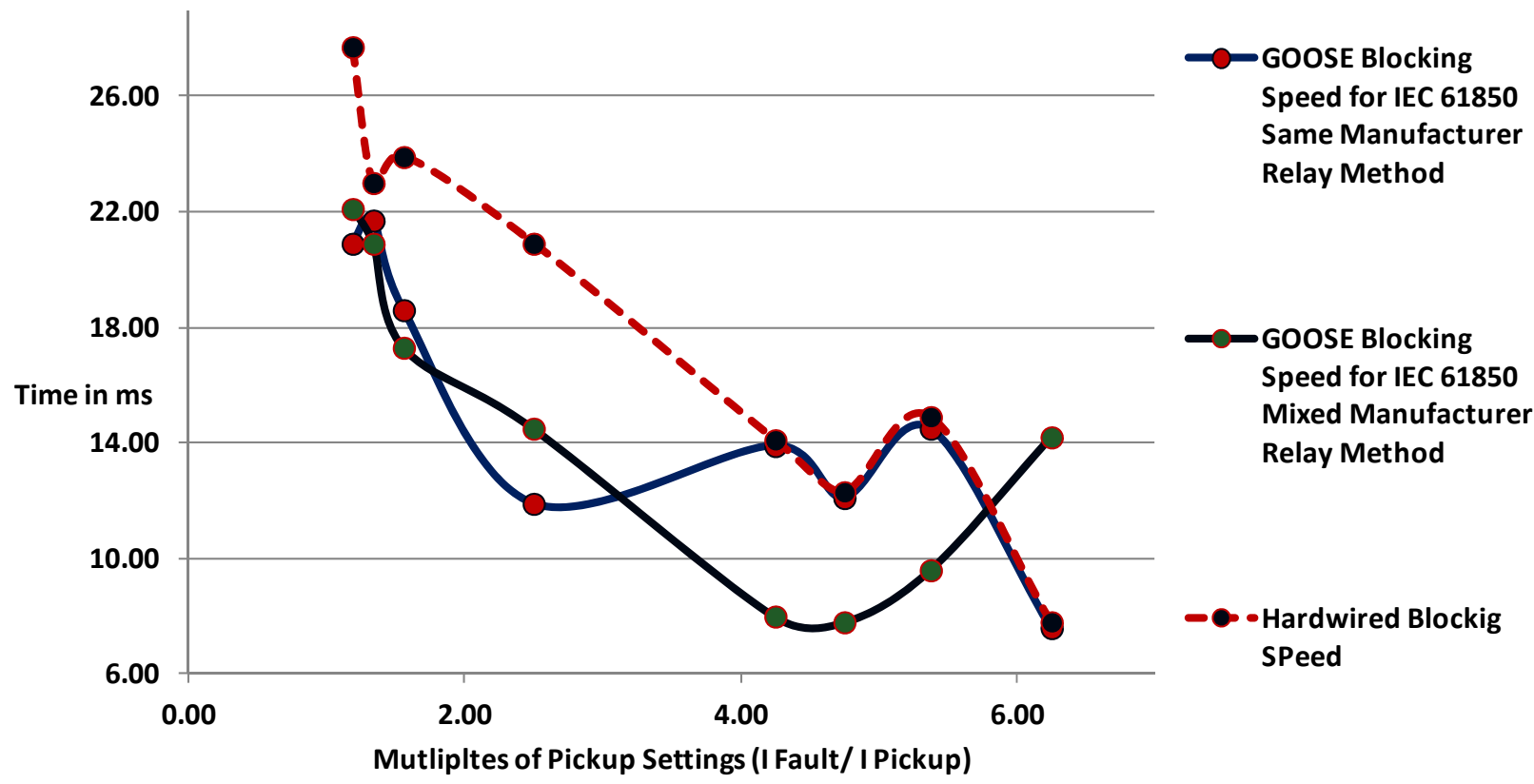

Figure 6.3 GOOSE Blocking Speeds Versus Hardwired Blocking Speed for Phase Faults

The test results show that for both ground faults and phase faults, the IEC 61850 method was not always faster than the hardwired method for fast bus protection scheme. This fact discredits that the IEC 61850 could improve system disturbance clearing time for the fast bus protection scheme. 
Interoperability of IEC 61850

Based on the collected data and the complexity in configuring the scheme, this study conveys that IEC 61850 GOOSE messaging is a viable replacement for the hardwired method for the fast protection scheme. The protection scheme worked well in both setups using relays from the same manufacturer and relays from different manufacturers. The average test results for the blocking speed using different manufacturers were within $4.0 \mathrm{~ms}$ of each other which was insignificant. These facts prove that interoperability is a feature of IEC 61850.

\section{Engineering and Construction Costs}

The results of the study support the claim that IEC 61850 can reduce the installation cost and lower maintenance cost. The experiments proved that no hard wired or a physical relay output contact was needed to detect for the out of zone faults. The experiments confirm that the high speed peer-to-peer GOOSE messages for each fault type of feeder relay(s) can be mapped to the virtual inputs of the bus relay. It was validated the bus relay received the blocking signals from the feeder relay(s) in an adequate time.

Although only two extra wires and a relay output contact of the feeder relay were needed to complete the hardwired fast bus protection scheme, rewiring must be done if a relay replacement is needed. The risk of disturbing energized equipment unintentionally cannot be ignored. Human errors not only cause interruptions to the power reliability but also might cause personal injuries, regardless of the complexity of any type of task being performed.

Figure 6.4 [22] illustrates the difference between the construction of a tradition hardwired substation and the construction of a new IEC 61850 type substation. The picture on the left shows more disorderly conditions of the relay back panels using the hardwired method. The 
picture on the right shows fewer wires are involved in the relay back panel. This is because virtual relay-to-relay communication is replacing the relay hard wiring circuits.

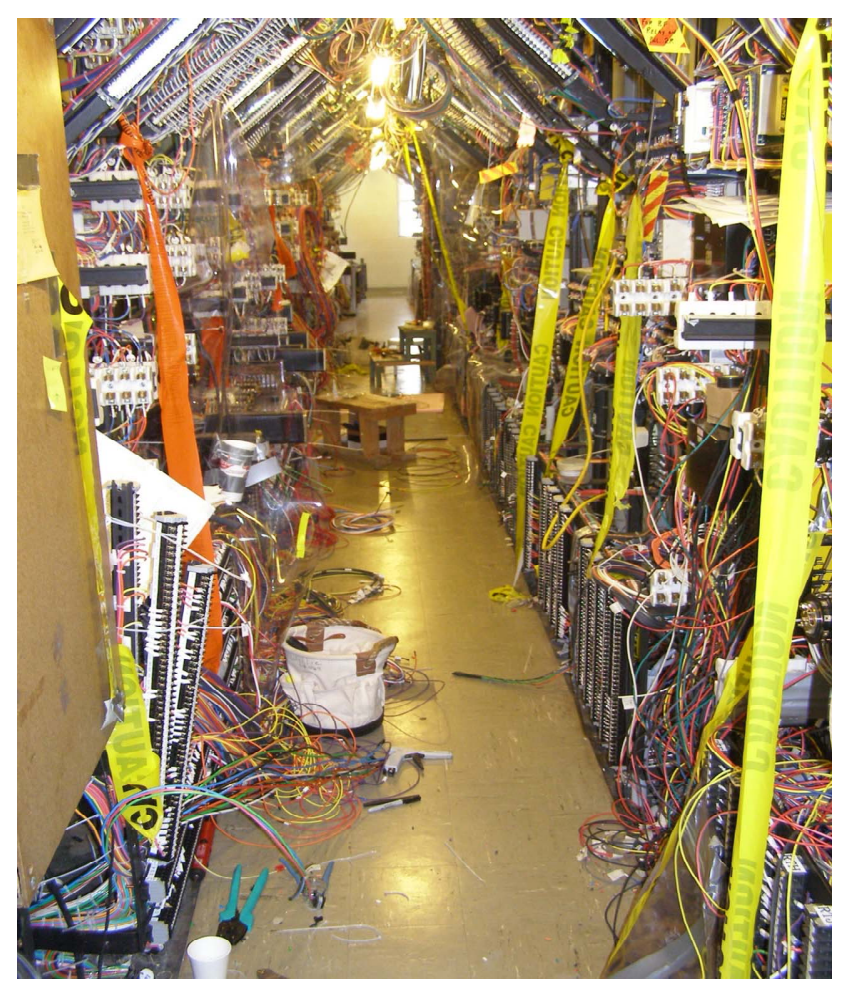

HARDWIRED METHOD (1965)

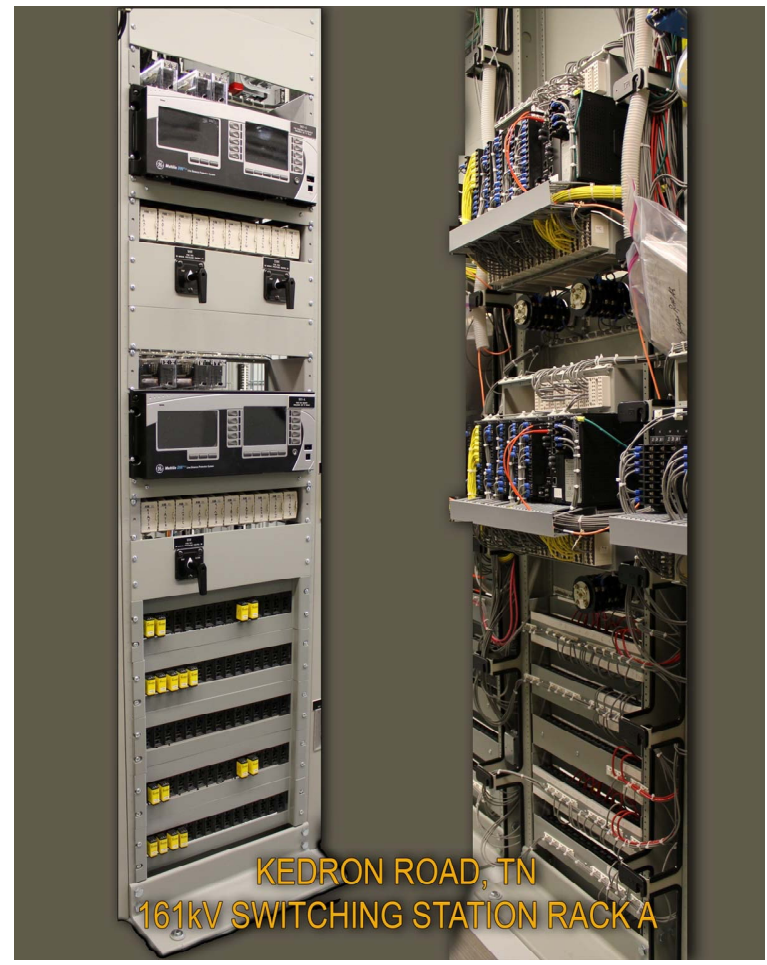

IEC 61850 METHOD (2012)

Fig 6.4 Pictures of Construction Using a Hardwired Method and an IEC 61850 Method 


\section{CHAPTER 7}

\section{CONCLUSIONS}

IEC 61850 GOOSE messaging offers compelling advantages especially for retrofitting, when compared to the traditional hardwired method, for the fast bus protection scheme. Although IEC 61850 GOOSE messaging offers more advantages in reducing engineering and construction costs, IEC 61850 does not have a significant improvement in the system disturbance clearing time. The results of this research show that IEC 61850 is a workable replacement for the traditional hardwired method for the fast bus protection scheme, but it is not an optimal solution. If relay protection speed is not crucial compared to installation cost, it will be an advantage to implement IEC 61850 for the fast bus protection scheme. The results of this study also show that if protection speed is the most important factor, conducting a special study before implementing the IEC 61850 method is recommended. IEC 61850 GOOSE interoperability was implemented successfully using relays of mixed manufactures in the study.

The IEC 61850 standard has essentially been developed for use within substations, but is now being seen as a key standard for a possible use of Smart Grid. As the computer and computer networking technology continue to expand and improve, the power industry is moving toward real time smart grid protection and automation control. IEC 61850 provides a vision of the future where the cost of the communications protocol is offset because it is more efficient and economical. Because of its international standard and its interoperability features, IEC 
61850 will eventually raise the effectiveness of the power grids around the world. IEC is proving itself to be the evolution of the communications protocol of the future. 


\section{REFERENCES}

[1] Daquing Hou and Dave Dolezilek, "IEC 61850 - What It Can and Cannot Offer to Traditional Protection Schemes", Schweitzer Engineering Laboratories, Inc., p. 8, p.9.

[2] Alex Apostolov, "Impact of IEC 61850 on Bus Protection", PACWORLD Magazine, December 2011 Issue, p. 42-43.

[3] Karl Zimmerman, "Microprocessor-Based Distribution Relay Applications", Schweitzer Engineering Laboratories, Inc., p. 3.

[4] Veselin Skendzic and Armando Guzman, "Enhancing Power System Automation Through the Use of Real-Time Ethernet”, Schweitzer Engineering Laboratories, p. 9-10.

[5] "Reducing Outage Durations through Improved Protection and Auto-restoration in Distribution Substations", Power System Relaying Committee, WG-K3, Final Draft 13.2, p. 5, September 2009.

[6] Craig Wester and Mark Adamiak, "Practical Applications of IEC 61850 Protocol in Industrial Facilities", GE Digital Energy Multilin, p. 1, 2.

[7] Klaus-Peter Brand, "IEC 61850 Short Tutorial, CIGRE, p. 13, 16.

[8] Grant Gilchrist, “IEC 61850”, May 2005, p.29.

[9] "Substation Automation Migration Strategies to IEC 61850 Using Relion Protection and Control", ABB, Automation and Power World, April 2011.

[10] Rick Hunt, Terrence Smith, Craig Wester, "Basics of IEC 61850 Protocol”, Georgia Tech Relaying Conference, May 2011, p. 6, p 52.

[11] Nelli Sichwart, "Transformer Load Tap Charger Control Using IEC 61850 GOOSE Messaging”, May 2012 Master Thesis, the University of Tennessee at Chattanooga, p. 4-5.

[12] IEC 61850 Protocol API User Manual, "Protocol Integration Stack", SystemCorp Pty LTD, p.4. 
[13] Hubert Kirrmann, "Introduction to The IEC 61850 Electrical Utility Communication Standard", ABB Switzerland Ltd, Corporate Research", p. 44.

[14] Craig Wester and Mark Adamiak, "Practical Applications of Ethernet in Substation and Industrial Facilities", GE Digital Energy Multilin, p.3, p. 9.

[15] Farel Becker, "IEC 61850 feeds grid protection and control", IntelligentUtility, Jan 24, 2013.

[16] Jose' Luis Ruiz, "Performance Comparison of A Permissive Overreach Transfer Tripp (POTT) Scheme Over IEC 61820 and Hard-Wire", May 2012 Master Thesis, the University of Tennessee at Chattanooga, p. 4-5.

[17] Tony Zhao, Lubomir Sevov, Craig Wester, "Advanced Bus Transfer and Load Shedding Applications with IEC 61850”, Texas A\&M 64th Relay Conference, April 13, 2011, p5.

[18] Craig Wester and Drew Baigent (GE), Juergen Holbach and Julio Rodridurez (Siemens), Lars Frisk and Steven Kunsman (ABB), and Luc Hossenlop (AREVA), "Status on the First IEC 61850 Based Protection and Control, Multi-Vendor Projects in the United States", 2007 60th Annual Conference for Protective Relay Engineers, March 2007.

[19] Schweitzer Engineering Laboratories, "SEL 751 Feeder Relay Instruction Manual", p. 2.20, p. 4.9 .

[20] GE Digital Energy Multilin, "D60 Line Distance Protection System Instruction Manual", p. 2-18.

[21] Prem K. Naik, Nirmal K-C Nair, and Valerity Vyatkin, "Sympathetic Trip Protection Scenario in IEC 61850", Univeristy of Auckland, New Zealand, p. 1.

[22] Different Pictures of Substations Built in 1965 and 2012, Courtesy of the Tennessee Valley Authority. 


\section{VITA}

Cassandra Ha Goff was born in Saigon, Vietnam. She came to the United States as a refugee. She worked her way through college and earned her bachelor degree in Electrical Engineering at Mississippi State University. She went to work for the Tennessee Valley Authority right after college where she met her husband, Mark.

Cassandra and her husband, Mark, decided to go back to pursue a master degree in Electrical Engineering at the University of Tennessee at Chattanooga(UTC) in Spring of 2011. Cassandra is currently working for the Tennessee Valley Authority as a system engineer and an adjunct professor for the Electrical Engineering Department at UTC. Cassandra is also a registered professional engineer in the state of Tennessee. 